\title{
Locus Coeruleus Activity Strengthens Prioritized Memories Under Arousal
}

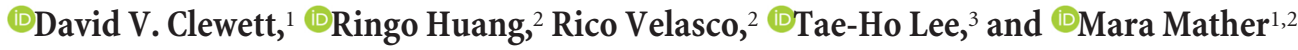 \\ ${ }^{1}$ Neuroscience Graduate Program, ${ }^{2}$ Davis School of Gerontology, University of Southern California, Davis, California 90089, and ${ }^{3}$ Department of Psychology \\ and Neuroscience, University of North Carolina at Chapel Hill, Chapel Hill, North Carolina 27599
}

Recent models posit that bursts of locus ceruleus (LC) activity amplify neural gain such that limited attention and encoding resources focus even more on prioritized mental representations under arousal. Here, we tested this hypothesis in human males and females using fMRI, neuromelanin MRI, and pupil dilation, a biomarker of arousal and LC activity. During scanning, participants performed a monetary incentive encoding task in which threat of punishment motivated them to prioritize encoding of scene images over superimposed objects. Threat of punishment elicited arousal and selectively enhanced memory for goal-relevant scenes. Furthermore, trial-level pupil dilations predicted better scene memory under threat, but were not related to object memory outcomes. fMRI analyses revealed that greater threat-evoked pupil dilations were positively associated with greater scene encoding activity in LC and parahippocampal cortex, a region specialized to process scene information. Across participants, this pattern of LC engagement for goal-relevant encoding was correlated with neuromelanin signal intensity, providing the first evidence that LC structure relates to its activation pattern during cognitive processing. Threat also reduced dynamic functional connectivity between high-priority (parahippocampal place area) and lower-priority (lateral occipital cortex) category-selective visual cortex in ways that predicted increased memory selectivity. Together, these findings support the idea that, under arousal, LC activity selectively strengthens prioritized memory representations by modulating local and functional network-level patterns of information processing.

Key words: emotion; locus ceruleus; memory; motivation; norepinephrine; pupil

\section{Significance Statement}

Adaptive behavior relies on the ability to select and store important information amid distraction. Prioritizing encoding of task-relevant inputs is especially critical in threatening or arousing situations, when forming these memories is essential for avoiding danger in the future. However, little is known about the arousal mechanisms that support such memory selectivity. Using fMRI, neuromelanin MRI, and pupil measures, we demonstrate that locus ceruleus (LC) activity amplifies neural gain such that limited encoding resources focus even more on prioritized mental representations under arousal. For the first time, we also show that LC structure relates to its involvement in threat-related encoding processes. These results shed new light on the brain mechanisms by which we process important information when it is most needed.

\section{Introduction}

Adaptive behavior relies on the efficient selection and storage of important information among competing inputs, particularly

\footnotetext{
Received July 25, 2017; revised Nov. 16, 2017; accepted Nov. 28, 2017.

Author contributions: D.V.C. and M.M. designed research; D.V.C., R.H., and R.V. performed research; D.V.C. contributed unpublished reagents/analytic tools; D.V.C., R.H., R.V., T.-H.L., and M.M. analyzed data; D.V.C., R.H., R.V., T.-H.L., and M.M. wrote the paper.

This work was supported by the National Institutes of Health (Grant R01AG025340) and by the University of Southern California (Endowed Fellowship to D.V.C.). We thank Dr. Alexa Tompary for helpful comments on the manuscript and Avi Chanales for assistance in editing one of the figures.

The authors declare no competing financial interests.

Correspondence should be addressed to David V. Clewett, Neuroscience Graduate Program, University of Southern California, 3715 McClintock Avenue, Room 351, Los Angeles, CA 90089. E-mail: clewett@usc.edu.

DOI:10.1523/JNEUROSCI.2097-17.2017

Copyright $\odot 2018$ the authors $\quad 0270-6474 / 18 / 381558-17 \$ 15.00 / 0$
}

under threatening or arousing conditions. Emotionally arousing stimuli are preferentially attended and encoded, often at the cost of processing surrounding neutral information (Strange et al., 2003; Hurlemann et al., 2005; Anderson et al., 2006; LaBar and Cabeza, 2006; Phelps et al., 2006; Kensinger et al., 2007; Knight and Mather, 2009; Bisby and Burgess, 2013). However, arousal's influence on cognition is not limited to enhancing emotional memoranda and suppressing neutral stimuli. Rather, arousal seems to enhance processing prioritized (e.g., goal-relevant) representations regardless of their emotionality while also suppressing less salient representations (Easterbrook, 1959; Mather and Sutherland, 2011; Sutherland and Mather, 2012; Sakaki et al., 2014).

For instance, emotional sounds or images enhance memory of goal-relevant neutral stimuli seen just previously (Sakaki et al., 
2014; Lee et al., 2015) and impair memory of less salient neutral information (Strange et al., 2003; Hurlemann et al., 2005; Sakaki et al., 2014; Clewett et al., 2017a). Similar memory enhancements have been reported in studies pairing shock with neutral images (Dunsmoor et al., 2012; Schwarze et al., 2012; Dunsmoor et al., 2015). Moreover, memory for neutral information benefits from the receipt of reward or punishment (Adcock et al., 2006; Murty et al., 2012; Weymar et al., 2013). An important open question is whether, like emotional stimuli, threat of punishment enhances memory for salient neutral stimuli by activating arousal-related brain mechanisms.

Although the dopaminergic system facilitates reward-motivated encoding (Adcock et al., 2006; Shohamy and Adcock, 2010), recent findings suggest that dopamine does not support encoding of neutral items incentivized by threat of punishment (Murty et al., 2012). Instead, threat-motivated encoding appears to be driven by the amygdala, a region that enhances emotional memories via norepinephrine (NE) modulation (McGaugh and Roozendaal, 2002; Strange and Dolan, 2004; McGaugh, 2013; Markovic et al., 2014). Further, the LC coordinates processing of a wide range of motivationally significant stimuli (Aston-Jones et al., 1999; Berridge and Waterhouse, 2003; Mohanty et al., 2008; Sara, 2009; Bouret and Richmond, 2015; Varazzani et al., 2015). Therefore, the LC is well equipped to amplify task-relevant encoding when it is most needed.

Theoretical models posit that phasic LC activity enhances neural gain such that strong inputs are further enhanced while weaker inputs are further inhibited (Usher et al., 1999; Aston-Jones and Cohen, 2005; Eldar et al., 2013). According to the recent "glutamate amplifies noradrenergic effects" (GANE) model, neural gain is achieved via local interactions between NE release and excitatory glutamate transmission, which signals the activation strength of a mental representation (Mather et al., 2016). In this framework, local NE-glutamate interactions upregulate excitation in regions transmitting important information, leading to more selective neuronal processing and memory outcomes. These local excitatory effects are also thought to engage lateral inhibitory processes that suppress weaker, competing inputs.

In this fMRI study, we tested GANE's core prediction that arousal recruits LC activity to enhance task-relevant memory representations and suppress task-irrelevant representations (Mather et al., 2016). During scanning, participants performed a monetary incentive encoding task in which they prioritized a background scene in attention and memory while ignoring a transparent foreground object. Sympathetic arousal was induced on some trials by threatening to deduct money from a preset account if participants later forgot loss-cued scenes. We hypothesized that pupil dilations, a biomarker of phasic LC activity (Murphy et al., 2014; Varazzani et al., 2015; Joshi et al., 2016; Reimer et al., 2016), would predict enhanced scene memory and impaired object memory. We also hypothesized that pupil dilation would selectively modulate successful scene encoding activity in LC and parahippocampal place area (PPA), a region specialized to process scenes (Epstein and Kanwisher, 1998), and queried whether such patterns were related to LC neuromelanin signal intensity (Keren et al., 2009). Finally, we investigated whether threat-related LC activity amplifies competition within relevant functional networks, yielding greater memory selectivity under arousal.

\section{Materials and Methods \\ Participants}

Thirty-two healthy young adults were recruited from the University of Southern California's Psychology Subject Pool and nearby community to participate in this experiment. All participants provided written informed consent approved by the University of Southern California Institutional Review Board and received monetary compensation for their participation and performance on the task. All eligible individuals had normal or normal-to-corrected vision and hearing and were not taking $\beta$-blockers or psychoactive drugs. To limit the influence of sex hormones (i.e., progesterone and estradiol) on the predicted memory and arousal effects, female participants had to be: (1) using monophasic hormonal contraceptives and (2) using those contraceptives for at least 3 months. Female participants only completed the experiment on their active pill days (days 8-21).

Six participants were excluded from data analysis: 2 participants fell asleep during the scan; 1 participant had excessive head movement during the scan $(>2 \mathrm{~mm}) ; 2$ participants violated recruitment criteria on the day of their scan; and 1 participant failed to follow task instructions. Because the focus of this study was on examining LC activity as indexed by pupil dilation, an additional four participants were excluded due to poor eye-tracking quality (e.g., pupil values were unattainable for $>50 \%$ of the encoding trials $)$. This resulted in 22 participants $\left(\mathrm{M}_{\text {age }}=22.18\right.$; $\mathrm{SD}_{\text {age }}=2.44 ; 10$ female) for all analyses.

\section{Monetary incentive encoding task \\ Stimuli}

The visual stimuli consisted of 96 neutral objects and 96 neutral scene images selected from previous datasets (Gabrieli et al., 1997; Kensinger et al., 2006) and the Internet. Objects consisted of animals or kitchen utensils centered on a white background. Half of the scene images depicted indoor scenes and the other half depicted outdoor scenes. Each of these images was resized to be $300 \times 300$ pixels and rendered in grayscale. From these images, 96 "overlap" stimuli were created by overlaying one object image on top of one scene image; the object image was rendered transparent using Adobe Photoshop 5.0 such that the foreground object and background scene images were equally discernable. Each of the grayscale, unmerged object and scene stimuli were yoked with a categorically similar but perceptually different image that served as a foil during the recognition memory test. This resulted in a total of 192 scene and object images.

To isolate cognitive-related changes in pupil dilation, image luminance was normed across all of the task screens (i.e., overlap stimuli, fixation crosses, scrambled images, and money symbols) using the SHINE toolbox in MATLAB (Willenbockel et al., 2010). To model an "active baseline" (Stark and Squire, 2001) in the fMRI analyses, scrambled overlap images were generated by shuffling a $0.27^{\circ} \times 0.27^{\circ}$ box in a random fashion. Threat cues were accompanied by a negative buzzer sound acquired from the International Affective Digitized Sounds (Sound ID \#712; $M_{\text {Arousal }}=$ $7.98, S D_{\text {Arousal }}=1.62 ; M_{\text {Valence }}=2.42, S D_{\text {Valence }}=1.62$; Bradley and Lang, 2007). No sound was paired with the neutral money cues.

\section{Task design}

fMRI data were acquired during the monetary incentive encoding task. The overall structure of the experiment was a 2 (priority: high vs low) $\times$ 2 (arousal: threat vs neutral) within-subjects design. There were a total of 96 encoding trials in the task, which were subdivided into four blocks of 24 trials each. Half of the trials in each block belonged to the threat condition and the other half to the neutral condition. Stimulus order was randomized across all blocks and the order of the arousal trials was randomized within each block.

\section{Encoding phase}

During the task, participants were presented with a series of grayscale "overlap" images of a transparent object overlaid on a background scene (Fig. 1). At the beginning of each trial, participants were shown 1 of 2 gray monetary symbols for $1 \mathrm{~s}$. In the threat condition, a "no symbol" cued participants that they would lose 50 cents if they forgot the upcoming scene on the subsequent memory test. In the neutral condition, a gray square symbol cued participants that, although they should still try to memorize the background scene, they would not be punished for forgetting it later. To increase arousal on threat trials, each threat cue was 


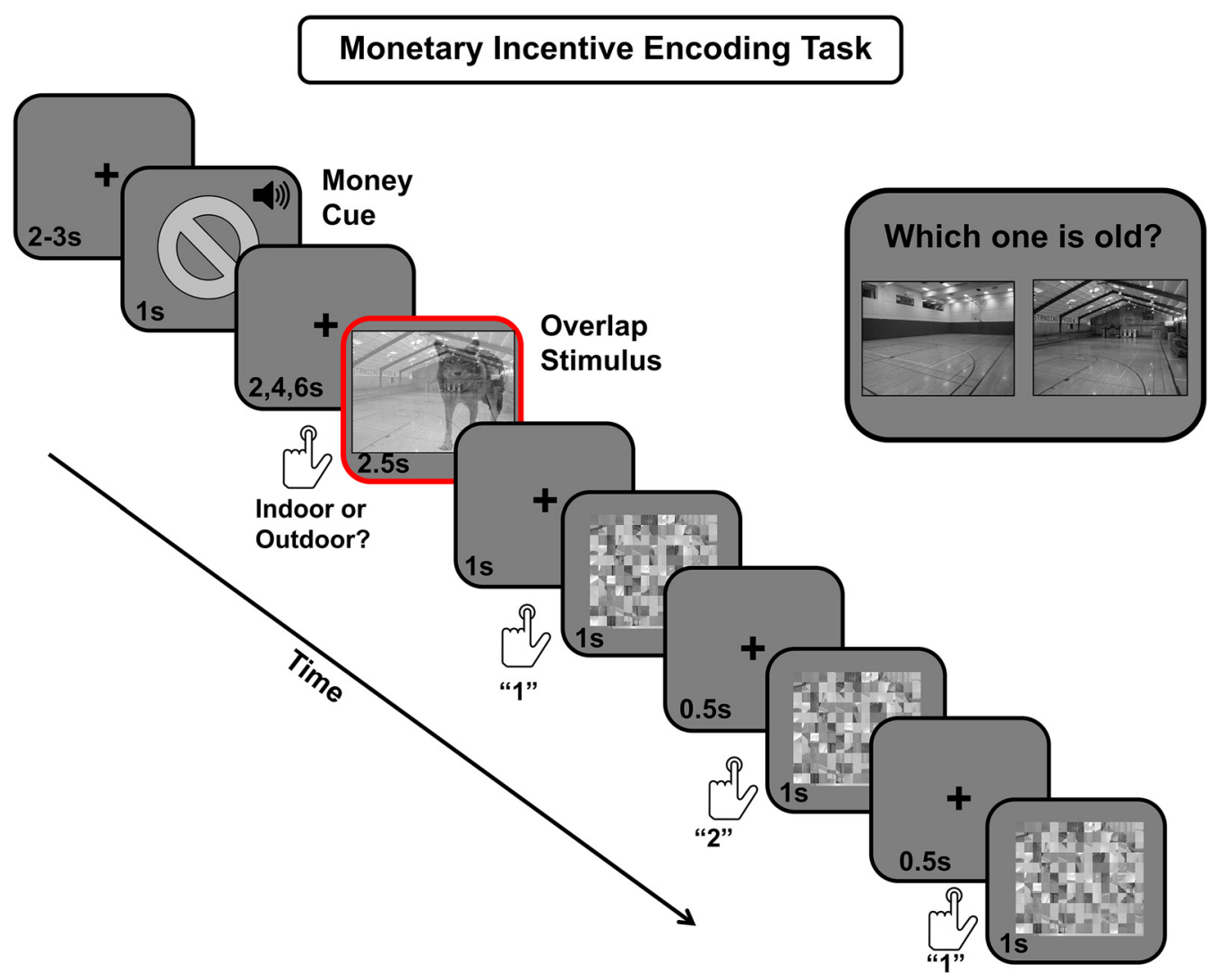

Figure 1. Schematic trial from the monetary incentive encoding fMRI task. Each trial consisted of a money cue, a jittered interstimulus interval, an overlap image (scene/object combo), and a distracter task. In this task, participants were instructed to prioritize each background scene in attention and memory while ignoring the transparent object. On half of the trials, participants were motivated to encode the scenes by a threat cue ("no symbol") accompanied by an arousing buzzer noise (threat condition). This symbol cued participants that they would lose 50 cents from a preset account if they forgot the upcoming scene on a subsequent recognition memory test. On the other half of trials (neutral condition), participants saw an image of a square. On these trials, they were instructed to try to memorize the scene as best as they could, but they had no additional incentive to do so. The red square denotes the $2.5 \mathrm{~s}$ stimulus-encoding period when brain activity was analyzed. The scrambled overlap images were used to model an "active baseline" in the fMRI analyses and to prevent participants from rehearsing recent scenes. The top right panel shows a schematic trial from the two-alternative forced choice memory test that was administered outside of the scanner $\sim 15$ min after encoding. Note that images are not drawn to scale.

accompanied by a highly arousing buzzer sound. The monetary cue was followed by a jittered central fixation cross $(2,4$, or $6 \mathrm{~s})$. After this variable anticipation period, the overlap image appeared for $2.5 \mathrm{~s}$.

To manipulate goal relevance participants were instructed to only focus on and memorize each scene in the overlap images while ignoring the foreground object. To reduce the salience/priority of the object even further, participants were told that their memory would not be tested for any of the objects and that they were only meant to be a distraction. To facilitate encoding and ensure participants were prioritizing the scenes, they were also instructed to categorize each scene as indoors or outdoors as quickly and accurately as possible when the overlap image appeared. Regardless of the cue, participants were instructed to categorize and memorize the exact scene image on each trial.

Following the overlap image, participants performed a brief scrambledimage detection task, which was used to: (1) prevent memory rehearsal of recent scenes and (2) provide an active baseline period that could be modeled in the neuroimaging analyses. In this task, participants saw 3 scrambled overlap images for $1 \mathrm{~s}$ each, which were separated by $1 \mathrm{~s}$ fixation cross screens in between. Participants had to respond with their right hand as quickly as possible when they saw each scrambled image. They pressed the left button when they saw the first scrambled image, the right button when they saw the second scrambled image, and the left button again for the third scrambled image. Each trial concluded with a $2-3 \mathrm{~s}$ jittered fixation cross, which served as the intertrial interval.

\section{Memory test}

Approximately $15 \mathrm{~min}$ after completing the monetary incentive encoding task in the scanner, participants were administered a two-alternative forced choice recognition memory test outside of the scanner (Fig. 1, top right). To examine the differential effects of threat-related arousal on memory for high versus lower priority images, we tested memory for all scene and object images that appeared during the task. There were 192 memory trials (96 of each visual category) in total. Before beginning the memory test, participants were told that their memory would be tested for all objects and scenes and to try their best on every trial.

On each memory trial, one object or scene image from the encoding task was displayed alongside a semantically similar but perceptually different (e.g., shape, orientation, etc.) foil image. The order of stimulus presentation was randomized and an equal number of old items appeared on the left and right of the screen. Participants had to indicate which of the two images they had seen in the scanner.

After each choice, participants were prompted to rate their confidence in their memory accuracy according to one of three options: extremely confident, somewhat confident, or just guessing. Participants had to make their memory responses within $10 \mathrm{~s}$ and their confidence rating within $5 \mathrm{~s}$. If they failed to respond within these response deadlines, the screen automatically advanced to the next trial and memory accuracy was recorded as a miss. To prevent fatigue, the memory test was divided into three blocks.

\section{Procedure}

Upon arriving for the experiment, participants provided written informed consent and completed several pen-and-paper questionnaires. Next, participants were given instructions for the monetary incentive encoding task on a computer. To familiarize participants with the exper- 
iment, they completed six task practice trials and three memory test practice trials before entering the scanner.

Scanning procedures took $\sim 1 \mathrm{~h}$. Inside the MRI scanner, participants first completed the PPA/lateral occipital cortex (LOC) functional localizer task (see the "PPA/LOC section" for details), which was followed by a T1-weighted high-resolution neuroanatomical scan. After the structural scan, participants completed four runs of the monetary incentive encoding task, which lasted $\sim 24 \mathrm{~min}$. Between each run, participants were reminded of the task instructions and to keep their head as still as possible. Scanning concluded with an $\sim 2$ min neuromelanin-sensitive weighted scan, which was used to help localize and delineate the LC in the brainstem in each participant (Shibata et al., 2006; Keren et al., 2009; Clewett et al., 2016).

After exiting the scanner and finishing the memory test, participants completed a custom postexperiment questionnaire about how arousing and unpleasant they found the buzzer sounds on threat trials and how arousing they found the threat and neutral money cues. All ratings were made on a 7 -point scale ranging from $1=$ not at all to $7=$ very (see Table 1). To assess punishment-related motivation, participants also indicated how motivated they were to memorize scenes on threat trials and neutral trials separately by making a vertical hash mark along a line spectrum ranging from "not motivated" on the left to "extremely motivated" on the right. Motivation responses were converted to scores by measuring the distance of the hash mark from the leftmost point (not motivated) in centimeters. In addition, to ensure that participants prioritized the scenes during the task, they were asked whether they tried to memorize all of the scenes and objects.

\section{MRI data acquisition}

All neuroimaging data were acquired on a 3T Siemens PRISMA scanner located at the University of Southern California Dana and David Dornsife Neuroimaging Center. The visual stimuli were displayed on a liquid crystal display monitor $(1024 \times 768$ pixels at $60 \mathrm{~Hz})$, which participants viewed via a mirror attached to a 32 -channel matrix head coil. A highresolution T1-weighted anatomical image (MPRAGE) was acquired to aid with functional image coregistration ( slices $=176$ axial; TR/TE $/ \mathrm{TI}=$ $2300 \mathrm{~ms} / 2.26 \mathrm{~ms} / 1060 \mathrm{~ms}$; FOV $=256 \mathrm{~mm}$; in-plane resolution $=1$ $\mathrm{mm}^{2}$; slice thickness $=1 \mathrm{~mm}$ with no gap; bandwidth $=200 \mathrm{~Hz} / \mathrm{Px}$; GRAPPA with acceleration factor $=2$; duration: 6 min and $45 \mathrm{~s}$ ).

Functional images for one run (190 volumes) of the localizer task and four runs (183 volumes each) of the monetary incentive encoding task were acquired using one echoplanar imaging sequence $(\mathrm{TR} / \mathrm{TE}=$ 2000/25 ms, 41 interleaved slices with no gaps, FOV = $192 \mathrm{~mm}$; FA = $90^{\circ} ; 3 \mathrm{~mm}$ isotropic voxel size). After the monetary incentive encoding task, we collected a neuromelanin-sensitive weighted MRI scan using a T1-weighted fast spin echo imaging sequence (TR/TE $=750 / 12 \mathrm{~ms}$, $\mathrm{FA}=120^{\circ}, 1$ average to increase SNR, 11 axial slices, FOV $=220 \mathrm{~mm}$, bandwidth $=220 \mathrm{~Hz} / \mathrm{Px}$, slice thickness $=2.5 \mathrm{~mm}$, slice gap $=3.5 \mathrm{~mm}$; in-plane resolution $=0.429 \times 0.429 \mathrm{~mm}^{2}$ ).

\section{Memory analyses \\ Mean task and memory performance}

To probe top-down attention during the monetary incentive encoding task, we performed paired $t$ tests on scene categorization accuracy and reaction times comparing threat and neutral trials. To determine how threat differentially influenced memory for high-priority and lowerpriority information, we performed a 2 (priority: high vs low) $\times 2$ (arousal: threat vs neutral) repeated-measures (rm)ANOVA. Planned two-tailed follow-up paired $t$ tests were also used to test whether, compared with neutral trials, threat enhanced memory for scenes and impaired memory for objects. Memory performance was calculated as the proportion of correctly remembered trials in each arousal trial type.

\section{Memory codependency analysis}

According to arousal-biased competition theory, arousal biases limited mental resources toward prioritized representations such that goalrelevant stimuli are even more likely to be remembered at the expense of remembering their competing distracters (Mather and Sutherland, 2011). Therefore, to determine how threat-induced arousal influenced competitive memory tradeoffs at the trial level, we performed a memory codependency analysis (Clewett et al., 2017a,b). Specifically, we investigated how memory accuracy for each scene differed according to memory accuracy for its corresponding object. Each of the 96 trials from the monetary incentive encoding task was categorized as one of four possible outcomes: (1) remembered scene and forgot object, (2) forgot scene and remembered object, (3) remembered both, or (4) forgot both. The frequencies of each memory outcome were calculated for threat and neutral conditions separately.

To test our main behavioral prediction that arousal enhances goalrelevant memory selectivity rather than memory more globally (e.g., remembering both the scene and its corresponding object), we performed a 2 (arousal: threat vs neutral) $\times 2$ (memory outcome: remembered $_{\text {scene }}$ forgot $_{\text {object }}$ vs remembered both) rmANOVA. Follow-up two-tailed paired $t$ tests were used to examine the main effects of arousal on selective versus global memory outcomes.

\section{Eye tracking}

Pupil dilation analysis. During fMRI scanning, pupil size was measured continuously at $60 \mathrm{~Hz}$ using an infrared ASL model 504 eye-tracker system (Applied Science Laboratories). To remove noisy or unreliable samples, pupil data were preprocessed using the following steps: (1) an upper and lower threshold for pupil diameter was applied by measuring participants' maximum pupil size while viewing a purely black screen and minimum pupil size while viewing a purely white screen; (2A) eye blinks and other artifacts were removed using a linear interpolation algorithm that constrained interpolation to periods of data loss $<1 \mathrm{~s}$; (2B) interpolation was applied $100 \mathrm{~ms}$ before the first missing sample and ended $100 \mathrm{~ms}$ after the last missing sample to account for rapid changes in pupil size during blinks; and (3) trials with estimated pupil dilations that were 3 SDs above or below the average pupil dilation were excluded from analysis.

Stimulus-evoked pupil dilation responses were examined for two events of interest: the money cue and the overlap stimulus. For the money cue, average pupil diameter was estimated from 1-2.5 $\mathrm{s}$ after cue onset. For the overlap stimulus, average pupil diameter was estimated across a $1 \mathrm{~s}$ window centered upon the time point of the maximum pupil size. The search window for the maximum pupil size was constrained to 1-2.5 s after scene onset, when the stimulus-evoked pupil response was most apparent. To measure dilation, average pupil size during these periods was baseline normed by subtracting the average pupil size during the $500 \mathrm{~ms}$ window before event onset. To examine the influence of threat-related arousal on pupil dilation, we performed two-tailed paired $t$ tests comparing average pupil dilation between threat and neutral trials during the cue and overlap stimulus periods separately.

Relationship between threat-evoked pupil responses and memory selectivity. To determine whether pupil dilation responses were associated with enhanced memory selectivity under threat, we performed hierarchical generalized linear modeling (HGLM) analyses using the glmer function in the lme4 library (Baayen et al., 2008). The parameters were estimated with the maximum likelihood method in R ( $\mathrm{R}$ Core Team, 2012). Each trial was used as a level 1 unit and each participant was used as a level 2 unit.

Arousal condition $(1=$ threat, $-1=$ neutral $)$ and mean pupil dilation responses to the overlap image were group centered and modeled as the level 1 predictors of trial-level memory outcomes. In two separate HGLMs, we examined how threat-induced pupil dilation responses to the overlap stimulus influenced scene (high priority) and object memory (lower priority) independently (dependent variables: $1=$ remembered scene/object; 0 = forgot scene/object). The main effects of arousal and overlap stimulus dilation and their interaction were included in these models. Next, we performed two more HGLMs to query whether dilations to the money cue interacted with dilations to the overlap stimulus to influence memory outcomes. These HGLMs was the same as before, but included an additional group-centered level 1 predictor for pupil dilation to the money cues. The main effects of arousal, overlap stimulus dilation, and money cue dilation were included in these models, along with all two- and three-way interactions. Additional HGLMs were performed to rule out the potential influence of other task or physiological 
predictors on memory, including baseline pupil size before the overlap image, scene memory confidence ratings, and anticipation duration between the money cue and overlap stimulus.

\section{Neuroimaging analyses \\ Image preprocessing}

Image preprocessing was performed using FSL Version 5.0.4 (fMRIB Software Library, www.fmrib.ox.ac.uk/fsl). The functional volumes were preprocessed according to the following steps: motion correction using MCFLIRT, removal of nonbrain tissue using BET, spatial smoothing using a Gaussian kernel of $6 \mathrm{~mm}$ full-width-at-half-maximum, grandmean intensity normalization of the entire $4 \mathrm{D}$ dataset by a single multiplicative factor, and a high-pass temporal filter of $100 \mathrm{~s}$. Structured noise and physiological artifacts, such as extreme head motion, white matter/ CSF signal, and cardiac pulsation/respiratory artifacts located across the whole brain, especially in the fourth ventricle, were identified and removed from the dataset using a single-session independent component analysis (ICA) (Beckmann et al., 2005). The criteria used for classifying noise components have been described in more detail previously (Clewett et al., 2013).

Next, two image registration procedures were used to query the effects of arousal on scene encoding at the whole-brain level. For the conventional alignment whole-brain analysis, each participant's denoised mean functional volume was coregistered to his or her T1-weighted highresolution anatomical image using brain-based registration. Anatomical images were then coregistered to the $2 \mathrm{~mm}$ isotropic MNI-152 standardspace brain using an affine registration with 12 degrees of freedom (DOF). For the brainstem-specific analysis, we performed an additional affine transformation with trilinear interpolation. In this step, registration was weighted by a binarized brainstem mask thresholded at $50 \%$ tissue-type probability (Harvard-Oxford Probabilistic Atlas). Prior research has shown that this technique is optimal for aligning voxels within the brainstem (Napadow et al., 2006).

\section{Pupil-parametric general linear models (GLMs) of subsequent memory effects}

Whole-brain analysis. Functional images were analyzed to determine how threat-induced arousal affected perceptual and successful encodingrelated activity across the whole brain using a parametric GLM. We created separate event-related regressors by modeling the onset times of the monetary cues and the overlap stimuli with durations of $1 \mathrm{~s}$ and $2.5 \mathrm{~s}$, respectively. Each task regressor was convolved with a dual-gamma canonical hemodynamic response function and their temporal derivatives were used to model data. Importantly, fMRI analyses were focused on the period when participants viewed the overlap image.

Trials were sorted by arousal type and scene memory outcome. A lower-level GLM was then constructed for each participant using four task regressors: (1) threat scene remembered, (2) threat scene forgotten, (3) neutral scene remembered, and (4) neutral scene forgotten. To examine how putative LC activity modulated brain activity encoding, four complementary task regressors were modeled to examine the parametric relationship between trial-level pupil dilation and brain activity during encoding. These parametric regressors were scaled according to the magnitude of the pupil dilation response to each overlap stimulus. As a result, the BOLD response was weighted more strongly on trials with larger stimulus-evoked pupil responses than on trials with smaller pupil responses, thereby providing an index of phasic noradrenergic modulation of local brain activity (Murphy et al., 2011). Pupil dilation values were mean-centered across each fMRI run and input into the "strength" column of the three-column format EV files in FSL.

Eight additional regressors were included in the GLM: two task regressors modeled activity during the money cue (threat and neutral) and six nuisance regressors derived from the motion correction procedure accounted for residual head motion. Three separate contrasts were created to test for main effects of memory (remembered vs forgot), arousal (threat vs neutral), and an arousal-by-memory interaction effect: [threat (remembered $>$ forgot) - neutral (remembered - forgot)]. Wholebrain statistical parametric brain maps were acquired separately for images registered using the conventional approach and brainstemoptimized approach.

From the lower-level statistical parametric maps, a second-level fixedeffects analysis was performed across each participant's functional runs. The resulting contrast images were analyzed in higher-level mixed-effects analysis using fMRIB's local analysis of mixed effects (FLAME $1+2$; (Beckmann et al., 2003). A single group average for each of the contrasts of interest was calculated using a one-sample $t$ test. To correct for multiple comparisons, $Z$-statistic images were thresholded using clusters determined by $Z>2.3$ and a corrected cluster significance threshold of $p=$ 0.05 (Worsley, 2001). In addition, this entire procedure was repeated for subsequent object memory outcomes to probe the specificity of arousal's effects on processing prioritized information.

PPA/LOC functional localizer. To examine how threat-induced arousal differentially influenced encoding-related activity in visual categoryselective cortex, we performed a functional localizer task to delineate the PPA (high-priority region) and LOC (lower-priority region) in the left and right hemisphere of each participant. The localizer scan consisted of 48 scrambled objects, 48 objects, and 48 scene images. Scrambled images of the intact stimuli were generated using the shuffling procedure described in the "Stimuli" section. All images were resized to $300 \times 300$ pixels, gray-scaled, luminance-normed and displayed on a gray background. Image presentation was divided into six blocks lasting $60 \mathrm{~s}$ each. These blocks were further subdivided into three miniblocks lasting $20 \mathrm{~s}$ each in which a series of images from one of the three visual categories was displayed on the screen (e.g., scrambled-only, object-only, or sceneonly). Each miniblock contained 8 images lasting $10 \mathrm{~s}$ and were separated from each other by a $10 \mathrm{~s}$ fixation cross. Each image was displayed for $1 \mathrm{~s}$, followed by $250 \mathrm{~ms}$ fixation cross interstimulus interval. The order of miniblocks was counterbalanced across participants. Participants were instructed to view the images passively as if they were watching a movie. Importantly, none of the stimuli from the localizer task were used in the monetary incentive encoding task.

\section{Region-of-interest (ROI) analyses for pupil-parametric GLM} Univariate PPA/LOC ROI analyses. ROI analyses were performed to determine how threat modulated successful scene and object encodingrelated activity in high-priority (PPA) and low-priority (LOC) visual category-selective cortex. The ROI masks for each PPA and LOC region were defined individually from the localizer session as 6-mm-radius spheres centered upon peak voxels in the ventral occipital and temporal cortex that were most selective for objects (object block $>$ scrambled objects + scene blocks; $Z=2.57$, uncorrected) and for scenes (scene block $>$ scrambled objects + object blocks; $Z=2.57$, uncorrected), respectively. Using this approach, the ROIs were definable for all participants for both LOC (mean peak MNI voxel coordinates: left [ $-42-71$ -8]; right [47 -69-8]) and PPA (mean peak MNI voxel coordinates: left $[-27-47-6]$; right $[27-44-9])$. These peak coordinates for the left and right PPA and LOC are displayed in standard space in Figure 6.

These functionally defined left/right PPA and left/right LOC masks were aligned to each participant's second-level statistical parametric maps and used to extract percentage signal change of task-related activity using FSL Featquery (www.fmrib.ox.ac.uk/fsl/feat5/featquery.html) To test our main hypothesis that successful scene encoding relates to enhanced activity in scene-selective visual cortex (PPA) and reduced activity in the lower-priority object-selective visual cortex (LOC) under arousal, we performed a 2 (region: PPA vs LOC) $\times 2$ (hemisphere: left vs right) $\times 2$ (arousal: threat vs neutral) $\times 2$ (memory: remember vs forgot) rmANOVA.

Univariate LC and ventral tegmental area (VTA) ROI analyses. A final ROI analysis for the LC was conducted using the same statistical procedures as above. To localize and delineate the LC in each individual, LC ROIs were hand drawn on each participant's neuromelanin-sensitive weighted image by two independent drawers using procedures described in a previous study (Clewett et al., 2016). The interrater reliability was 
high (intra-class coefficient [ICC] range: 0.85-0.99). Bilateral LC ROIs were manually defined as a $\sim 1.29 \mathrm{~mm}$ wide by $\sim 1.29 \mathrm{~mm}$ high (i.e., $3 \times$ 3 voxels) in an axial slice located $7 \mathrm{~mm}$ below the inferior boundary of the inferior colliculus. These masks were centered on the left and right voxels with the highest signal intensities neighboring the corners of the fourth ventricle. A dorsal pontine tegmentum (PT) reference ROI was defined as a $10 \times 10$ voxel square located 6 voxels above the more ventral of the 2 LCs and equidistantly between them.

We were unable to delineate LCs in 6 participants due to a scan not being collected $(n=2)$ or interspace registration issues $(n=4)$. For those participants, we used a standard-space 2 SD LC mask derived from a previous study (Keren et al., 2009). Each participant's LC ROI transformed into MNI $2 \mathrm{~mm}$ standard space using a slight variation of the transformation steps used for the whole-brain brainstem-weighted alignment procedure because the neuromelanin images had higher resolution. Neuromelanin-to-standard-space transformation steps included: (1) registering each participant's neuromelanin scan to his/her high-resolution anatomical scan using an affine transformation with 6 DOF; (2) registering each participant's high-resolution anatomical scan to the MNI standard $1 \mathrm{~mm}$ brain template using an affine transformation with 12 DOF; (3) performing an additional registration by weighting the T1-to-MNI $1 \mathrm{~mm}$ registration by a binarized MNI $1 \mathrm{~mm}$ standard-space mask; and (4) registering the MNI $1 \mathrm{~mm}$ standard-space brain to the MNI $2 \mathrm{~mm}$ standard-space brain to acquire a more sensitive, intermediate registration before transforming the LC ROIs to standard space. LC percentage signal changes values were extracted and submitted to a 2 (arousal: threat vs neutral) $\times 2$ (memory: remember vs forgot) rmANOVA to examine the effects of threat on successful scene and object encoding activity.

Although the VTA does not appear to support threat-motivated encoding processes (Murty et al., 2012), there are some indications that it can be activated by threat cues more broadly (Knutson et al., 2000; Shigemune et al., 2014). Therefore, to determine whether the dopaminergic system contributes to threat-enhanced memory selectivity, we also performed a VTA ROI analysis on scene and object encoding activity. To define this ROI, we thresholded and binarized a probabilistic VTA anatomical mask from a prior study (Murty et al., 2014) at 50\% and extracted percentage signal change values. These values were then submitted to the same rmANOVA analysis as the LC ROI.

\section{PPA functional connectivity during encoding}

Whole-brain analysis. To examine dynamic interactions between the PPA (high-priority visual cortex) and the rest of the brain during encoding, we performed a psychophysiological interaction (PPI) analysis (Friston et al., 1997). The same lower-level GLM was used as before, but included nine additional regressors (one physiological regressor and eight interaction regressors). Whole-brain PPI analyses were performed for the left and right PPA for object and scene subsequent memory effects separately.

The PPA physiological regressor was created aligning each participant's left and right PPA to his/her preprocessed functional data and extracting their mean activity time series. Eight interaction regressors were used to model the interaction between the PPA time series regressor and the pupil-modulated (parametric) and non-pupil-modulated (uniform scale) task-related regressors. As before, we tested for significant main and interaction effects of arousal (threat vs neutral) and memory (remember vs forgot) on whole-brain left and right PPA functional connectivity patterns modulated by trial-level changes in pupil dilation to the overlap stimulus. We applied the same multiple comparison corrections as the whole-brain univariate GLM.

PPA functional connectivity ROI analysis. Based on our a priori prediction that arousal amplifies competitive functional interactions between the PPA and LOC (Lee et al., 2014), we also performed ROI analyses. Using the same protocol as the whole-brain univariate GLM, each participant's left and right LOC was used to extract parameter estimates of left and right PPA connectivity from their conventionally registered whole-brain maps. The resulting parameter estimates were submitted to a 2 (priority: high vs low) $\times 2$ (PPA hemisphere: left vs right $) \times 2$ (LOC hemisphere: left vs right) $\times 2$ (arousal: threat vs neutral) $\times 2$ (memory: remember vs forgot) rmANOVA. To optimize PPA connectivity esti- mates with the LC, each participant's LC mask was used to extract parameter estimates from their brainstem-aligned maps. These PPA-LC ROIs were then submitted to the same statistical analyses as the LOC region but without an LC laterality factor.

\section{Individual differences in brain-behavior relationships}

In our final analyses, we queried the relationships between arousal's effects on local and network-level brain processing, LC structure (i.e., CNR), and threat-related memory selectivity across all participants using a Theil-Sen estimator, a robust linear correlation technique that is less sensitive to outliers than parametric regressions (Wilcox, 2004). To this end, we first computed threat-neutral difference scores for: (1) scene memory, (2) object memory, and (3) the difference between the two (i.e., scene neutral; gain effect in memory).

These three threat-related memory measures were then linearly correlated with LC contrast-to-noise values, LC and VTA ROI estimates, and PPA-LOC functional connectivity estimates.

Relationship between LC functional activation and structure. First, we examined whether LC signal contrast-to-noise ratio estimates, a putative biomarker for LC neuronal density and structural integrity (Keren et al., 2009, 2015), were associated with individual differences in LC-related scene and object encoding activity. LC contrast-to-noise ratios were calculated based on the mean LC signal intensity relative to the reference PT signal intensity using the following formula: $\mathrm{LC}_{\mathrm{CNR}}=\left(\mathrm{LC}_{\text {intensity }}-\right.$ $\left.\mathrm{PT}_{\text {intensity }}\right) / \mathrm{PT}_{\text {intensity }}$ (Sasaki et al., 2006; Shibata et al., 2006; Clewett et al., 2016). The two raters' left and right side estimates of LC contrast-tonoise ratios were all averaged together to increase the signal-to-noise ratio.

Four participants were excluded from this LC structure-function correlation analysis: two participants had striping artifacts in their neuromelanin images that overlapped with the LC and two other participants did not have neuromelanin scans due to time constraints. Of the remaining 18 participants with valid LC signal intensities, the overall mean and SD for LC contrast-to-noise ratio $(\mathrm{M}=0.19, \mathrm{SD}=0.045)$ was comparable to values in prior work using this technique (Clewett et al., 2016). Two robust correlations were performed between LC CNR values and threat-related LC scene encoding activity and object encoding activity separately.

Relationship between LC/VTA functional activation and memory. In the second robust regression analysis, we performed three robust regressions between the threat-related noradrenergic and dopaminergic encoding activity and memory: (1) threat-enhanced scene memory, (2) threatimpaired object memory, and (3) the subtraction between the two (i.e., scene effect - object effect; "gain effect" in memory). LC and VTA ROI estimates were modeled as predictors in the same robust regression to control for multiple comparisons ( $p=0.05$, two-tailed).

Relationships between threat-related PPA functional connectivity during successful encoding and memory. In the last regression analyses, we performed the same three brain-behavior correlations as the LC/VTA ROI analysis but using the PPA-LOC functional connectivity ROIs as predictors of memory performance. As before, to control for multiple comparisons, all six PPA-LOC connectivity combinations (two PPA hemispheres, two LOC hemispheres, and two item priority types) were modeled as brain predictors of threat-impaired object memory, threatenhanced scene memory, and their difference (scene - object subtraction) in three separate robust regressions (significance level: $p=0.05$, two-tailed).

\section{Results \\ Task performance}

Arousal/motivation ratings and encoding results

As expected, participants rated the threat money cues as being significantly more arousing than neutral money cues $\left(t_{(21)}=\right.$ 7.25, $p<0.001)$. Participants also indicated being significantly more motivated to memorize scenes on threat trials compared with neutral trials $\left(t_{(21)}=4.02, p=0.001\right)$ (Table 1). Other 
statistical comparisons from paired $t$ tests for attention accuracy (scene categorization), reaction times, and detection task performance are also displayed in Table 1.

\section{Memory performance}

Mean memory performance results

To determine whether threat-related arousal differentially influences memory of high-and lower-priority information, we performed a 2 (priority: high vs low) $\times 2$ (arousal: threat vs neutral) rmANOVA. Participants remembered significantly more goalrelevant scenes than distracting objects $\left(F_{(1,21)}=62.33, p<\right.$ $0.001, \eta_{\mathrm{p}}^{2}=0.75$; Fig. 2 , top). Consistent with our main behavioral prediction, threat-induced arousal had opposing effects on memory for high- and lower-priority stimuli $\left(F_{(1,21)}=15.32, p=\right.$ $0.001, \eta_{\mathrm{p}}^{2}=0.42$ ), with threat selectively enhancing high-priority memories. Planned comparisons indicated that this arousal-bypriority interaction effect on memory was predominantly driven by significant threat-induced enhanced memory of high-priority scenes $\left(t_{(21)}=4.26, p<\right.$ $0.001)$. In contrast, there was no significant threat-induced suppression of memory of low-priority objects $\left(t_{(21)}=-1.18\right.$, $p=0.25)$. However, separate one-tailed, one-sample $t$ tests revealed that object memory was significantly above chance on neutral trials $\left(t_{(21)}=3.31, p=0.003\right)$, but not on threat trials $\left(t_{(21)}=1.55, p=\right.$ $0.14)$, suggesting that floor effects for object memory in the threat condition may have limited the range for a meaningful statistical comparison.

\section{Memory codependency results}

To determine how threat-related arousal differentially influences memory selectivity at the trial level, we performed a 2 (memory outcome: remembered scene $_{\text {for- }}$ got $_{\text {object }}$ vs remembered both) $\times 2$ (arousal: threat vs neutral) rmANOVA. Overall, participants were significantly more likely to remember both stimuli than to remember the scene and forget its corresponding object $\left(F_{(1,21)}=6.30, p=0.02, \eta_{\mathrm{p}}^{2}=0.23\right.$; Fig. 2, bottom). There was no significant interaction effect on trial frequency.

Planned paired $t$ tests revealed that threat significantly enhanced memory for scenes at the cost of memory for competing objects $\left(t_{(21)}=3.69, p=0.006\right)$, whereas threat had no effect on global successful memory for paired scene and object stimuli $\left(t_{(21)}=0.90, p=0.38\right)$. In addition, the results indicated that participants were more likely to remember sceneobject pairs than to show a scene-biased memory tradeoff on neutral trials $\left(t_{(21)}=-2.70, p=0.013\right)$. Therefore, greater memory selectivity under threat appeared to be driven by less narrowing of attentional and encoding resources to neutral scenes.

\section{Pupil results}

Event-related pupil dilation

Planned two-tailed paired $t$ tests revealed that pupil dilations were significantly larger in response to money cues on threat compared
Table 1. Monetary incentive encoding task performance and post-task ratings of money cue arousal and motivation

\begin{tabular}{lccl}
\hline & Threat trials & Neutral trials & Significance \\
\hline Arousal rating for money cue & $3.95(1.36)$ & $1.59(0.091)$ & $p<0.001^{* * *}$ \\
Motivation to memorize scenes & $5.05(1.99)$ & $2.52(2.16)$ & $p=0.001^{* *}$ \\
Scene categorization accuracy & $0.94(0.08)$ & $0.94(0.05)$ & n.s. \\
Scene categorization RT & $1181.56(206.41)$ & $1136(139.08)$ & n.s. \\
Scrambled image \#1 accuracy & $0.91(0.09)$ & $0.90(0.08)$ & n.s. \\
Scrambled image \#2 accuracy & $0.96(0.04)$ & $0.96(0.05)$ & n.s. \\
Scrambled image \#3 accuracy & $0.95(0.05)$ & $0.97(0.04)$ & n.s. \\
Scrambled image\#1 RT & $557.18(77.87)$ & $542.86(81.29)$ & n.s. \\
Scrambled image \#2 RT & $325.02(52.19)$ & $318.05(46.54)$ & n.s. \\
Scrambled image \#3 RT & $324.2(48.48)$ & $319.98(48.25)$ & n.s.
\end{tabular}

Motivation ratings for memorizing the scenes were measured in centimeters from 0 (not motivated) to 7 (extremely motivated). Cue arousal ratings were measured on a scale from 1 (not at all) to 7 (very). $p$-values from paired $t$ test are displayed in the rightmost column comparing threat versus neutral trials.

RT, Reaction time; n.S., nonsignificant.

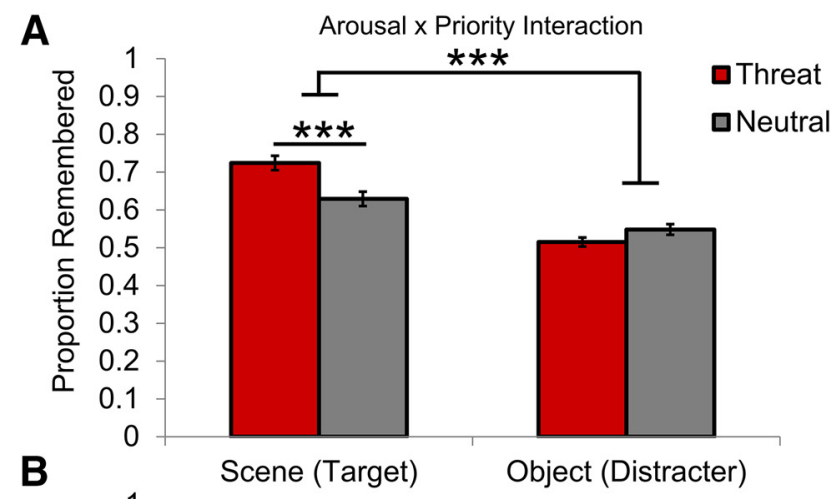

Figure 2. Memory performance on the monetary incentive encoding fMRI task. $\boldsymbol{A}$, Proportion of correctly remembered highpriority scenes and lower-priority objects by arousal condition (threat of punishment vs neutral). Data are represented as means \pm SEM. Statistical chance-level performance is 0.5 . $\boldsymbol{B}$, Left, Proportion of trials in which participants showed a selective memory trade-off in favor of the high-priority scene (left bars) or remembered both the scene and object stimuli in the overlap images (right bars). Right, Schematic of memory codependency analysis. Displayed object/scene images represent successful memory. Light gray squares signify bins there were used to calculate memory selectivity. ${ }^{* *} p<0.01,{ }^{* *} p<0.001$.

with neutral trials $\left(t_{(21)}=10.53, p<0.001\right.$; Fig. $\left.3 C\right)$. Conversely, pupil dilations were significantly larger in response to overlap images on neutral trials compared with threat trials $\left(t_{(21)}=\right.$ $-2.17, p=0.041$; Fig. $3 C)$.

Relationship between encoding-related pupil dilation and memory Next, we performed HGLM analyses to query the relationship between threat-related pupil dilation and high- and lower-priority stimulus memory outcomes. The HGLM analyses on scene memory revealed a significant main effect of arousal condition $(z=$ 4.57, $p<0.001$ ) on scene encoding, mirroring the results of the ANOVA. There was also a significant pupil dilation-by-condition 
A Pupil Responses to Money Cue

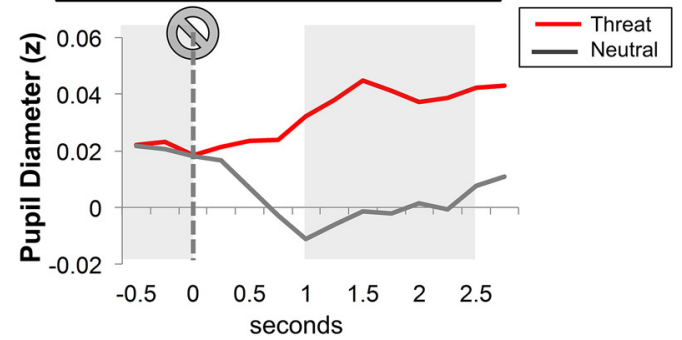

B Pupil Responses to Overlap Image

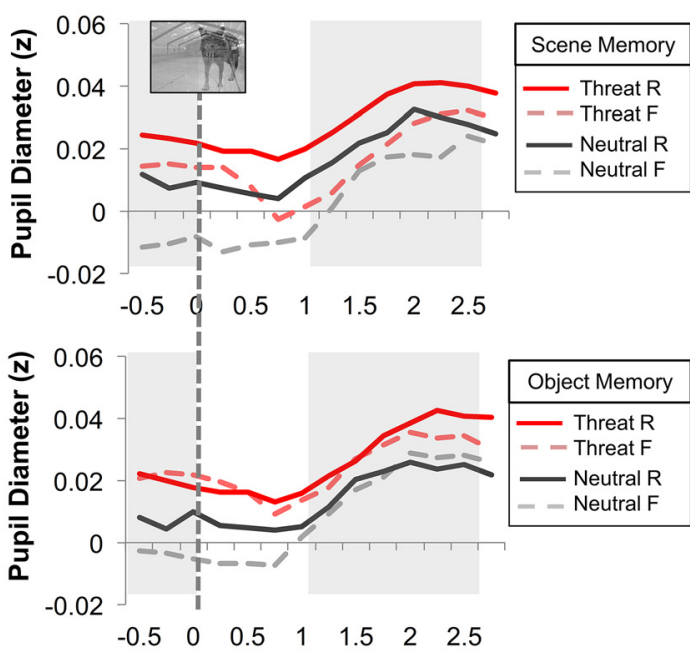

\section{Average Stimulus-Evoked Pupil Dilation}

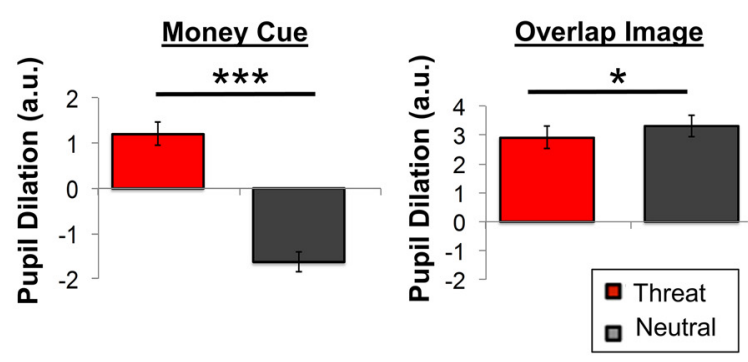

D Pupil-Memory Relationship for Scene Stimuli

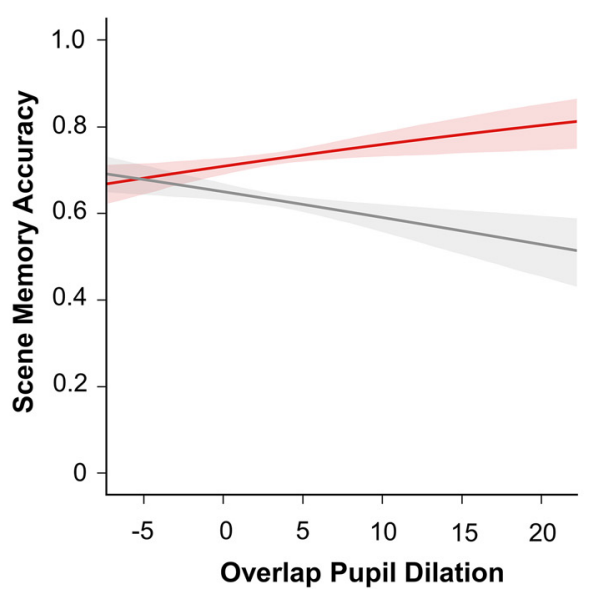

Figure 3. Pupil results. $\boldsymbol{A}$, Mean time course of evoked pupil diameter changes to the money cue broken down by arousal type (threat vs neutral). $\boldsymbol{B}$, Mean time course of evoked pupil diameter changes to the overlap stimulus broken down by memory type (scene vs object), arousal type (threat vs neutral), and memory outcome (remember vs forgot). In $\boldsymbol{A}$ and $\boldsymbol{B}$, pupil dilations were calculated by subtracting the window of peak dilation (rightmost shaded rectangles) minus pupil size during the baseline period (leftmost shaded rectangle). $\boldsymbol{C}$, Average pupil dilation was significantly larger in response to the threat money cue compared with the neutral money cue, but significantly lower in response to overlap images in the threat compared with the neutral condition. Data are represented as means \pm SEM. $\boldsymbol{D}$, Results from the HGLM analyses indicated that threat-enhanced pupil dilations to the overlap images predicted better scene memory compared with the neutral condition. Lines and shading indicate regression fits and SEs for the threat (red) and neutral (gray) conditions. The $y$-axis represents the probability of accurate scene recognition. Although not displayed, there were no significant effects for object memory outcomes. ${ }^{*} p<0.05$; ${ }^{* *} p<0.001$. R, Remember; $\mathrm{F}$, forgot.

Table 2. Hierarchical generalized linear modeling (HGLM) results examining the relationship between pupil dilation responses to the overlap stimulus and arousal condition (threat or neutral) on scene/object memory

\begin{tabular}{lrlll}
\hline Predictors & Estimate & SE & $z$ & $p$ \\
\hline $\begin{array}{l}\text { Scene memory } \\
\quad \text { Intercept }\end{array}$ & 0.77741 & 0.08815 & 8.819 & $<2.00 \mathrm{E}-16^{* * *}$ \\
$\quad$ Arousal condition & 0.22457 & 0.04914 & 4.57 & $4.87 \mathrm{E}-06^{* * *}$ \\
$\quad$ Overlap pupil dilation & 0.01611 & 0.01401 & 1.149 & 0.2505 \\
$\quad$ Vverlap dilation $\times$ condition & 0.03092 & 0.01405 & 2.2 & $0.0278^{*}$ \\
$\begin{array}{l}\text { Object memory } \\
\text { Intercept }\end{array}$ & 0.14405 & 0.04493 & 3.206 & $0.00135^{* *}$ \\
$\quad$ Arousal condition & -0.04403 & 0.04493 & -0.98 & 0.32719 \\
$\quad$ Overlap pupil dilation & -0.01391 & 0.01276 & -1.09 & 0.27592 \\
$\quad$ Overlap dilation $\times$ condition & 0.01076 & 0.01276 & 0.843 & 0.39929 \\
\hline
\end{tabular}

Arousal condition is coded as threat $=1$ and neutral $=-1$. Scene/object memory are coded as remember $=1$ and forgot $=0$.

${ }^{*} p<0.05 ;{ }^{* *} p<0.01 ;{ }^{* * *} p<0.001$

interaction effect on scene encoding $(z=2.20, p=0.028$; Table 2). Plotting this interaction effect revealed that threat-evoked pupil dilations during the overlap image predicted better scene encoding (Fig. 3D). There were no significant effects of pupil dilation on object memory, indicating that the beneficial mnemonic effects of arousal are unique to the high-priority stimulus $(p>0.05)$.
Several additional HGLMs were performed to rule out the potential influence of other task predictors on scene/object memory. In the first HGLM, we found that the overlap dilationby-condition interaction effect on scene memory remained significant after including a trial-level predictor for pupil dilations to the money cue and its interactions with other predictors (arousal condition and overlap dilation; $p<0.05$ ). There were no significant main or interaction effects of money cue dilations on scene or object memory. Next, modeling trial-level covariate predictors for cue-to-overlap anticipation duration and memory confidence rating did not alter the results in both the model with money dilations and without it (scene interaction effect: $p<0.05$; all object effects: $p>0.05$ ). There was, however, a significant main effect of scene memory confidence rating on successful scene encoding $(p<0.001)$.

We also created an HGLM model that included a term and interactions for average baseline pupil size before the onset of the overlap image based on suggestions that baseline pupil diameter predicts gain effects in learning (Eldar et al., 2013). Including these terms slightly diminished the overlap-by-condition interaction $(p=0.095)$, but there was no significant main effect or interactions of baseline pupil diameter on scene memory $(p>$ 0.46). Finally, in the object HGLM, there was significant main effect of anticipation duration on object memory such that longer waiting periods between the money cue and the overlap image 
Table 3. Effects of threat and object/scene memory encoding on whole-brain activity as parametrically modulated by pupil dilation responses to the overlap image

\begin{tabular}{|c|c|c|c|c|c|c|}
\hline \multirow[b]{2}{*}{ Brain region } & \multirow[b]{2}{*}{ Cluster } & \multirow[b]{2}{*}{ Z-statistic } & \multicolumn{3}{|c|}{ MNI peak coordinates (mm) } & \multirow[b]{2}{*}{ Voxels } \\
\hline & & & $x$ & $\gamma$ & $Z$ & \\
\hline \multicolumn{7}{|l|}{ Scene memory } \\
\hline \multicolumn{7}{|l|}{ Conventional cortical alignment } \\
\hline Forgot $>$ Remember & - & - & - & - & - & - \\
\hline Left frontal operculum & 3 & 3.67 & -40 & 16 & 2 & 1450 \\
\hline Left insula & 3 & 3.54 & -38 & 8 & -6 & \\
\hline Left inferior frontal gyrus & 3 & 3.29 & -62 & 26 & 12 & \\
\hline Left central opercular cortex & 3 & 3.29 & -44 & -6 & 8 & \\
\hline Left angular gyrus/supramarginal gyrus & 2 & 3.55 & -56 & -50 & 18 & 954 \\
\hline Left anterior supramarginal gyrus & 2 & 3.34 & -62 & -28 & 34 & \\
\hline Left middle temporal gyrus & 2 & 3.26 & -56 & -52 & 8 & \\
\hline Left posterior supramarginal gyrus & 2 & 3.21 & -60 & -50 & 10 & \\
\hline Right anterior supramarginal gyrus & 1 & 3.22 & 66 & -28 & 34 & \\
\hline Right anterior supramarginal gyrus & 1 & 3.2 & 62 & -28 & 46 & \\
\hline Right anterior supramarginal gyrus & 1 & 3.2 & 62 & -32 & 46 & \\
\hline \multicolumn{7}{|l|}{ Threat (Remember $>$ Forgot) - neutral (Remember $>$ Forgot) } \\
\hline Left lingual gyrus/PHG & 1 & 3.98 & -14 & -42 & -6 & 1127 \\
\hline Left parahippocampal gyrus & 1 & 3.84 & -14 & -40 & -10 & \\
\hline Right lingual gyrus/PHG & 1 & 3.81 & 14 & -40 & -10 & \\
\hline Left posterior hippocampus & 1 & 3.39 & -22 & -34 & -8 & \\
\hline Right temporal occipital fusiform cortex & 1 & 3.34 & 32 & -56 & -12 & \\
\hline Right temporal occipital fusiform cortex & 1 & 3.29 & 24 & -58 & -14 & \\
\hline \multicolumn{7}{|l|}{ Brainstem alignment } \\
\hline Threat $>$ neutral & - & - & - & - & - & - \\
\hline Remember $>$ Forgot & - & - & - & - & - & - \\
\hline \multicolumn{7}{|l|}{ Conventional cortical alignment } \\
\hline \multicolumn{7}{|l|}{ Remember $>$ Forgot } \\
\hline Right frontal pole & 2 & 3.35 & 26 & 46 & 20 & 938 \\
\hline Right precentral gyrus & 2 & 3.21 & 54 & 8 & 42 & \\
\hline Right frontal pole & 2 & 3.21 & 34 & 46 & 16 & \\
\hline Right inferior frontal gyrus & 2 & 3.18 & 56 & 14 & 32 & \\
\hline Right frontal pole & 2 & 3.16 & 30 & 46 & 14 & \\
\hline Right frontal pole & 2 & 3.08 & 32 & 50 & 24 & \\
\hline Right occipital pole & 1 & 3.5 & 10 & -94 & 16 & 843 \\
\hline Right occipital pole & 1 & 3.25 & 10 & -98 & 18 & \\
\hline Right precuneus cortex & 1 & 3.1 & 8 & -58 & 8 & \\
\hline Left intracalcarine cortex & 1 & 3.09 & -6 & -66 & 8 & \\
\hline Right precuneus cortex & 1 & 3.08 & 10 & -58 & 16 & \\
\hline Right superior lateral occipital cortex & 1 & 3.06 & 16 & -82 & 20 & \\
\hline Threat (Remember $>$ Forgot) - neutral (Remember $>$ Forgot) & & & & & & \\
\hline Right inferior lateral occipital cortex & 1 & 3.39 & 36 & -70 & 8 & 851 \\
\hline Right cerebellum & 1 & 3.23 & 38 & -60 & -24 & \\
\hline Right cerebellum & 1 & 3.2 & 36 & -58 & -28 & \\
\hline Superior lateral occipital cortex & 1 & 3.15 & 40 & -88 & 22 & \\
\hline Right cerebellum & 1 & 3.08 & 38 & -64 & -24 & \\
\hline Right occipital fusiform gyrus & 1 & 3.03 & 34 & -62 & -12 & \\
\hline Brainstem alignment & & & & & & \\
\hline Remember $>$ Forgot & - & - & - & - & - & - \\
\hline Threat (Remember $>$ Forgot) - neutral (Remember $>$ Forgot) & - & - & - & - & - & - \\
\hline
\end{tabular}

For brainstem alignment, results are only reported if clusters were centered upon voxels in brainstem or midbrain because this alignment procedure may lead to misalignment of higher subcortical and cortical structures. H, Hemisphere; PHG, parahippocampal gyrus; TO, temporal occipital; R, remember; F, forgot. 


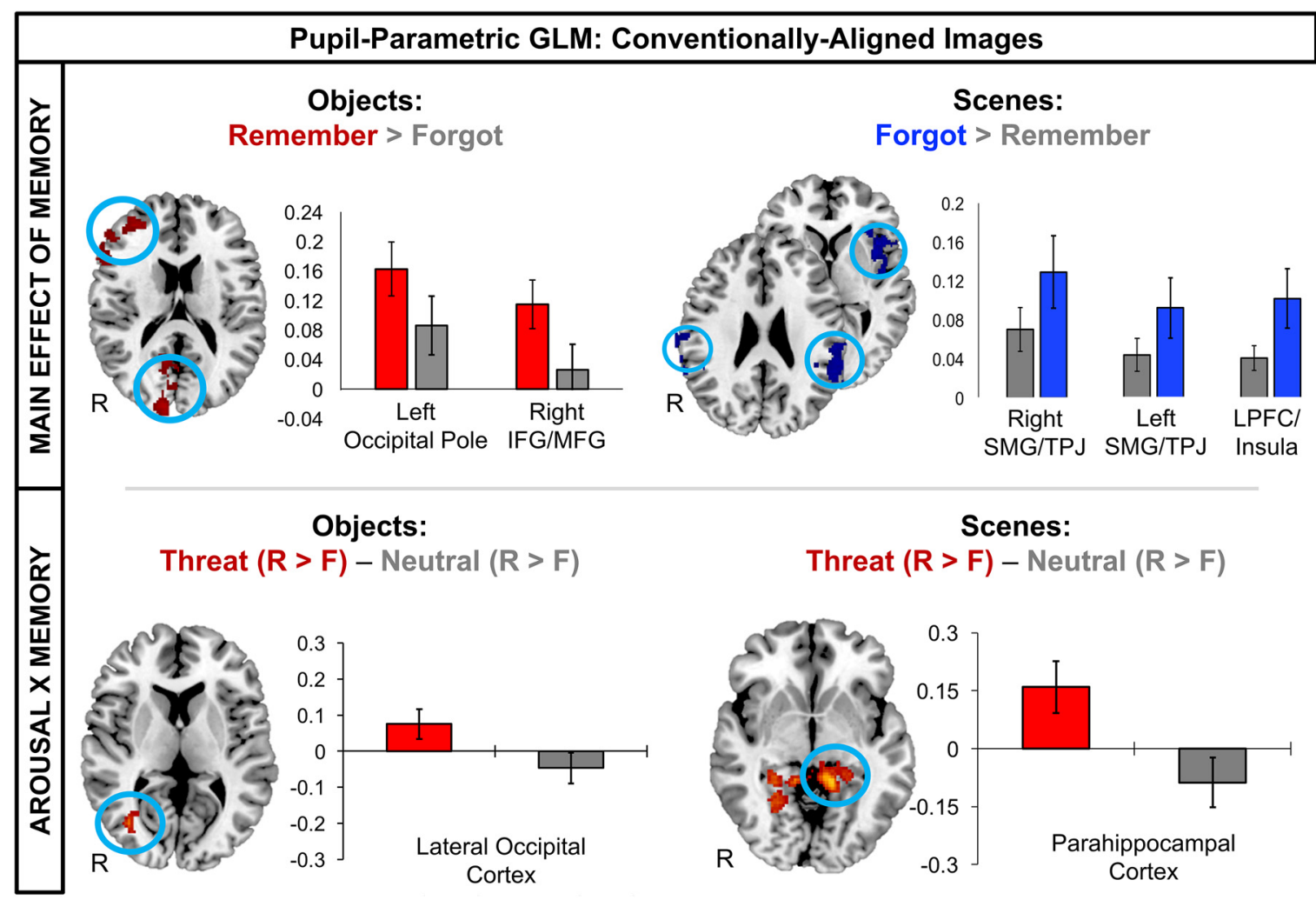

Figure 4. Whole-brain pupil-parametric GLM examining the effects of threat-induced arousal on scene and object encoding activity. Successful object encoding was associated with increased activity in the occipital pole and right inferior frontal gyrus (IFG) and middle frontal gyrus (MFG; top left). Successful scene encoding was associated with suppressed activity in left inferior and middle frontal gyrus (IFG/MFG), left insula, and bilateral TPJ encompassing the supramarginal gyrus (SMG; top right). Compared with the neutral trials, threat of punishment significantly enhanced object-encoding activity in the LOC (bottom left). Compared with the neutral trials, threat of punishment significantly enhanced scene-encoding activity in PHC (bottom right). Note that the statistical results are not displayed because the bar graphs are meant to illustrate the direction of the effects in each whole-brain cluster. Data are represented as mean percentage signal change \pm SEM. $R>F$, Remember > forgot; L, left; R, right.

was associated with worse object memory regardless of arousal condition $(z=-2.21, p=0.027)$.

\section{Brain results}

The neuroimaging results are organized into two main sections: the pupil-parametric GLM and the PPA-seeded functional connectivity analyses. Each of these sections was then broken down into three subsections: ROI results, whole-brain results, and brain-behavior correlations.

\section{Pupil-parametric GLM}

Catecholamine and visual-category-selective cortex ROI results. First, we performed parametric GLM analyses in which we scaled trial-level BOLD response to the overlap image by the magnitude of pupil dilation. To determine how threat-induced arousal affected scene and object encoding activity in the LC, VTA, and category-selective visual cortex ROIs, we extracted percentage signal change values from each participant's LC mask, as well as his/her left/right PPA and LOC that were identified by the functional localizer.

Consistent with a neural gain effect in memory, threatenhanced LC encoding activity significantly differed between trials with subsequently remembered scene and object stimuli (three-way interaction: $F_{(1,21)}=4.87, p=0.039, \eta_{\mathrm{p}}^{2}=0.19$ ). Follow-up arousal $\times$ memory ANOVAs revealed that this three-way interaction effect was driven by LC activity being significantly greater when participants encoded threat-motivated scenes compared with neutral-trial scenes $\left(F_{(1,21)}=6.56, p=\right.$ $0.018, \eta_{\mathrm{p}}^{2}=0.24$; Fig. 5 , Table 3$)$. In contrast, LC activity did not differ between threat and neutral conditions when participants successfully encoded distracter objects $(p>0.94)$. We did not find any significant main or interaction effects of arousal, priority, or memory on VTA activity, thereby ruling out a significant dopaminergic influence on competitive encoding processes under threat.

For the visual-category-selective cortex ROIs, there was no significant main effect of hemisphere on brain activity during encoding of scenes or objects $(p>0.05)$. Further, collapsing across hemispheres revealed no other main or interaction effects on PPA or LOC activity for scenes or objects $(p>0.05)$.

Whole-brain results. Analysis of pupil-modulated BOLD signal changes at the whole-brain level indicated that there was no significant main effect of arousal on brain activity during the overlap image. For scene encoding, there was a significant main effect of memory on brain activity, with successful scene encoding being associated with reduced activity in left frontal opercular cortex/insula, and bilateral supramarginal gyrus around the temporoparietal junction (TPJ). Supporting our hypothesis, there was a significant arousal-by-memory interaction effect on scene encoding activity such that, compared with the neutral condition, threat of punishment modulated successful scene encoding activity in the left posterior parahippocampal cortex (PHC), left posterior hippocampus, and left lingual gyrus (Fig. 4, Table 3).

For object encoding, we identified a significant main effect of memory on object encoding activity such that successfully encoding object stimuli corresponded with increased activation of right occipital pole and right lateral prefrontal cortex, including infe- 


\section{Pupil-Parametric GLM: Brainstem-Aligned Images}

Scenes: Threat (Remember > Forgot) - Neutral (Remember > Forgot)
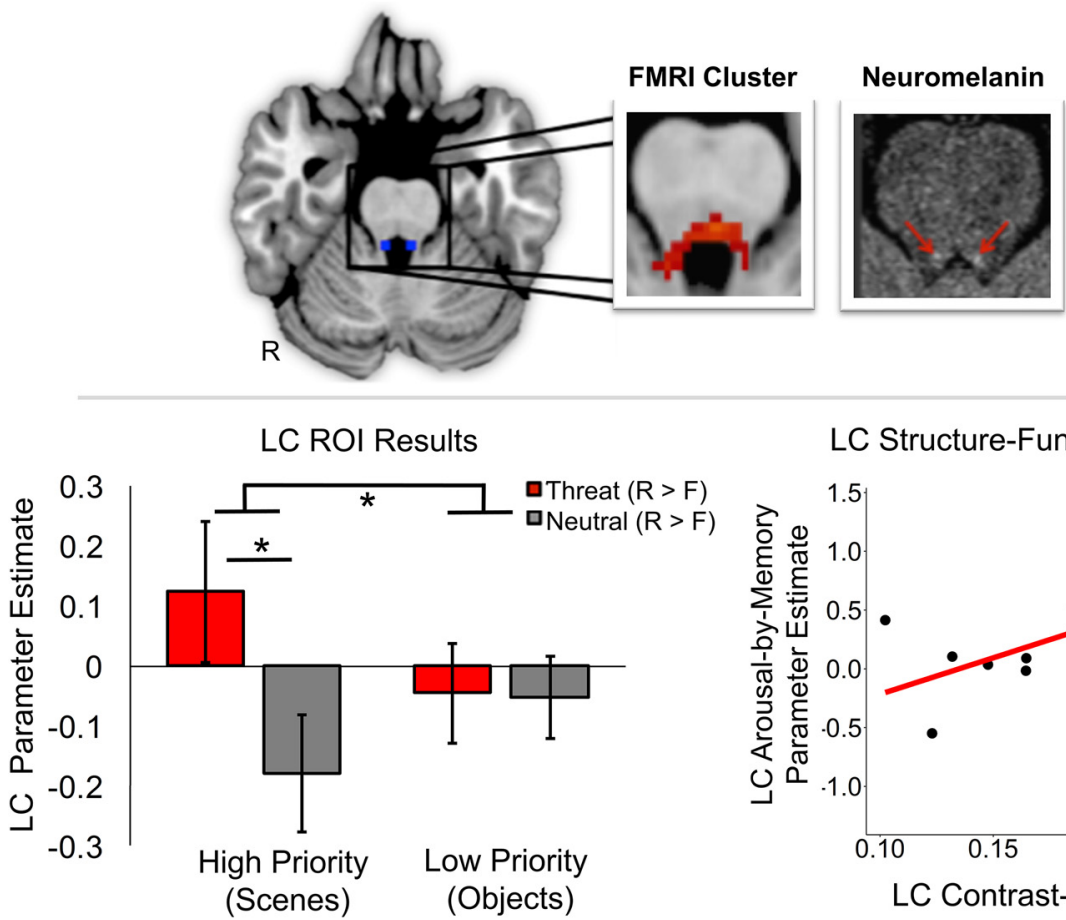

LC Structure-Function Relationship

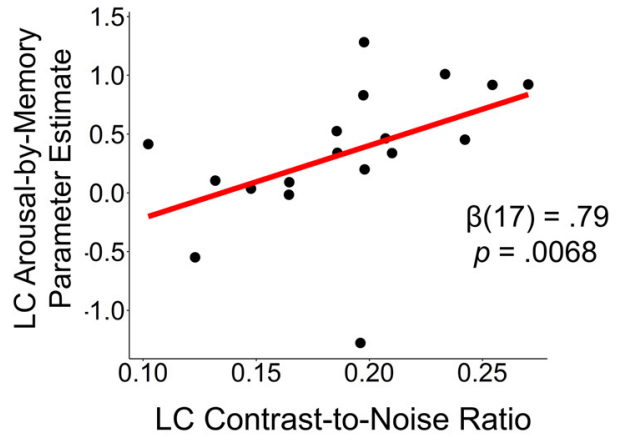

Figure 5. Threat of punishment amplified scene-encoding activity in the LC. In this pupil-parametric GLM analysis, participant's whole-brain statistical parametric maps were written into standard space using a procedure optimized for brainstem alignment (Napadow et al., 2006). Whole-brain analyses revealed that threat-enhanced scene encoding was associated with higher activity in a brainstem region encompassing the LC. Qualitative comparisons revealed substantial overlap between the spatial location of this functional cluster (red) and both the Keren et al. (2009) 2 SD $2 \mathrm{~mm}$ standard-space LC mask (blue dots) and one participant's neuromelanin-sensitive weighted image (bright dots; top). This result was confirmed to be the LC in a planned ROl analysis in which participants' hand-drawn neuromelanin $\mathrm{LC}$ masks were used to extract percentage signal change (bottom left). Data are represented as means \pm SEM. The degree to which participants engaged the $L C$ during threat-related scene encoding was also correlated with his/her $L C$ neuromelanin contrast-to-noise ratio (CNR), a putative biomarker of $L\left(\right.$ structure (bottom right). ${ }^{*} p<0.05$. $R$, Scene remembered; $F$, scene forgot.

rior frontal gyrus and middle frontal gyrus (Fig. 4, Table 3). We also found a significant arousal-by-memory interaction effect such that threat led to enhanced object encoding activity in a right LOC region (Fig. 4, Table 3 ).

In the brainstem-aligned images, threat of punishment modulated successful scene encoding activity in a brainstem/cerebellum cluster encompassing the known anatomical location of the LC (Fig. 5). There were no significant interactions or main effects on brain activity for object memory, suggesting that brainstem neuromodulatory nuclei did not significantly influence distracter encoding.

Relationship between individual differences in LC structure, LC function, and threat-related memory outcomes. Next, we investigated whether individual differences in LC structure, as indexed by the neuromelanin contrast-to-noise ratio, were related to LC ROI estimates of threat-enhanced scene encoding and threatimpaired object encoding from the prior analysis. We also investigated whether these LC structural and functional measures were associated with memory outcomes. Robust regression analyses revealed that, across participants, the degree of threat-enhanced LC scene encoding activity was significantly positively correlated with LC contrast-to-noise ratio $\left(\beta_{(21)}=0.79, p=0.0067\right.$; Fig. 5). Threat-related LC activity for object encoding was not significantly associated with LC contrast-to-noise ratio across participants $\left(\beta_{(21)}=0.60, p=0.15\right)$. This finding suggests that participants with a greater lifetime of LC engagement and concomitant NE metabolism (Mather et al., 2017), as signified by greater LC neuromelanin
Table 4. Robust multiple-regression results showing relationship between threat-related encoding activity in noradrenergic and dopaminergic ROIs for scenes/objects separately and threat-related memory outcomes

\begin{tabular}{lccc}
\hline Threat $(\mathrm{R}>\mathrm{F})>$ neutral (R $>$ F) ROl activity & Estimate & SE & $p$ \\
\hline Relationship with threat-related suppression of low-priority object & memory & \\
Intercept & -0.21 & 0.38 & 0.76 \\
Locus ceruleus: scene encoding & 0.07 & 0.38 & 0.93 \\
Ventral tegmental area: scene encoding & 0.41 & 0.32 & 0.18 \\
Locus ceruleus: object encoding & -0.20 & 0.32 & 0.69 \\
Ventral tegmental area: object encoding & -0.10 & 0.34 & 0.64 \\
Relationship with threat-related enhancement of high-priority scene & memory & \\
Intercept & 0.04 & 0.33 & 0.82 \\
Locus ceruleus: scene encoding & 0.26 & 0.42 & 0.41 \\
Ventral tegmental area: scene encoding & 0.09 & 0.28 & 0.82 \\
Locus ceruleus: object encoding & -0.65 & 0.29 & $0.08 \sim$ \\
Ventral tegmental area: object encoding & 0.16 & 0.35 & 0.82 \\
Relationship with threat-enhanced gain effects in memory & & \\
Intercept & 0.05 & 0.36 & 0.89 \\
Locus ceruleus: gain effect & 0.54 & 0.32 & 0.11 \\
Ventral tegmental area: gain effect & 0.17 & 0.28 & 0.39 \\
\hline
\end{tabular}

"Gain effect" refers to a two-way interaction score between priority, memory, and arousal: scene [threat $(R>F)-$ neutral $(\mathrm{R}>\mathrm{F})-\operatorname{object}[$ threat $(\mathrm{R}>\mathrm{F})-$ neutral $(\mathrm{R}>\mathrm{F})]$. $\sim p<0.10$.

$R$, Remember; F, forgot.

signal intensity, also activated the LC to a larger degree when forming goal-relevant memories under threat. There were no significant correlations between LC contrast-to-noise ratio and either memory outcome under threat $(p>0.05)$. 


\section{Functional Connectivity Results with Brain Region (PPA) Representing Prioritized Stimulus}

Scenes:

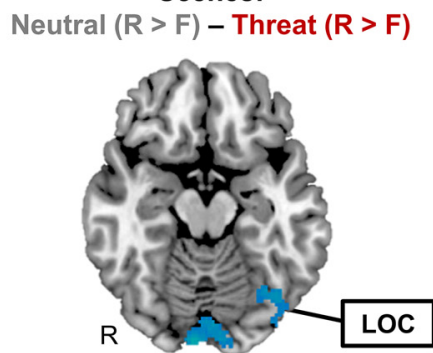

$p=.046^{*}$

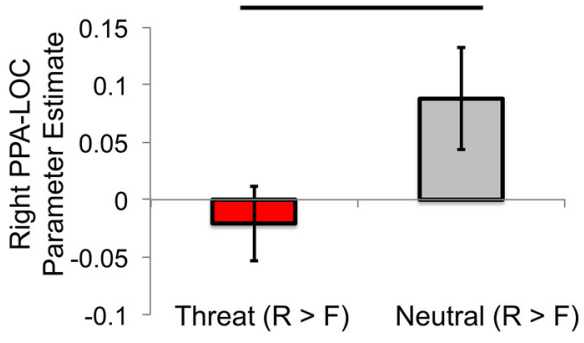

ROI Results

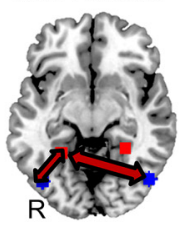

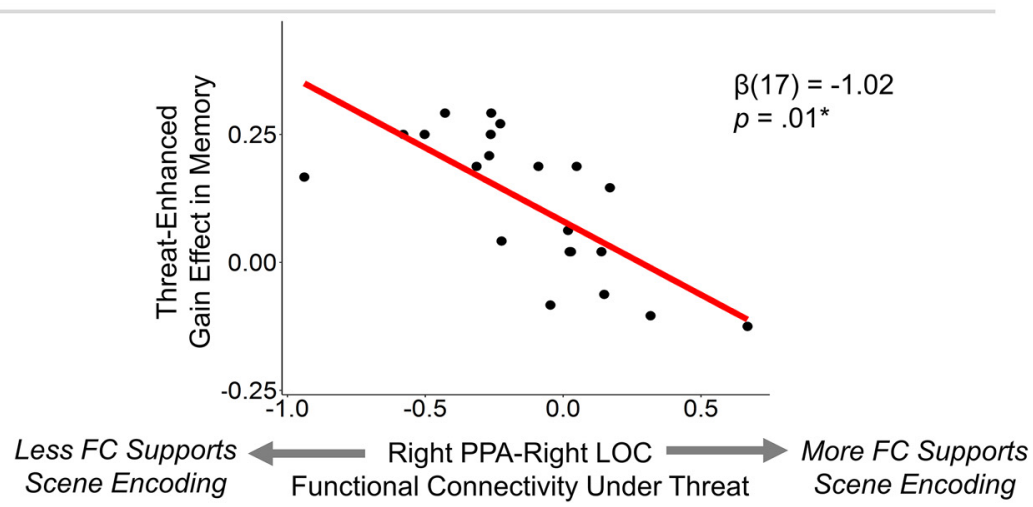

Figure 6. Dynamic functional connectivity between the PPA and the rest of the brain during scene encoding. At the whole-brain level, enhanced scene-encoding activity in the threat versus neutral condition was associated with reduced right PPA functional coupling with early visual processing cortical regions, including the left LOC (blue cluster; top left). ROI analyses using each participant's functionally defined LOC masks confirmed this result (top right). Data are represented as means \pm SEM. Right PPA functional connectivity with the LOC was associated with different memory outcomes under threat-induced arousal (bottom). Specifically, those who showed less right PPA-right LOC functional coupling during successful scene versus object encoding under threat also exhibited greater threat-related gain effects in declarative memory. Note that the statistic reflects the residual correlation coefficient after controlling for the relationship between gain effects in memory and the other PPA-LOC functional connectivity predictors. FC, Functional connectivity. ${ }^{*} p<0.05$.

When examining the relationship between threat-related LC and VTA object/scene encoding activity, we did not find any significant correlations with threat-related memory outcomes (Table 4). However, there was a statistical trend toward a significant correlation between LC gain-related encoding activity and enhanced memory selectivity under threat $\left(\beta_{(20)}=0.54, p=0.11\right)$.

PPA functional connectivity results

$R O I$ results. PPI analyses were used to determine whether arousal conditions and trial-level pupil dilations to the encoding stimulus modulated dynamic functional connectivity patterns between the PPA and LC. We did not find a significant main effect of priority $(p>0.05)$ or main effect of LOC side on brain activity ( $p$ s $>0.05)$. Therefore, we performed follow-up 2 (memory: remember vs forgot) $\times 2$ (arousal: threat vs neutral) ANOVAs collapsed across LOC sides and for object and scene memory separately.

For the scene encoding PPI analysis, we found that threat significantly reduced right PPA-LOC functional connectivity when scenes were successfully encoded $\left(F_{(1,21)}=4.50, p=0.046\right.$, $\eta_{\mathrm{p}}^{2}=0.18$; Fig. 6 , Table 5). This finding suggests that greater arousal-biased competition between high- and lower-priority visual cortical regions is associated with better goal-relevant memory under threat.

For the object encoding PPI analysis, there were no significant main or interaction effects of arousal and memory on PPA-LOC functional connectivity $(p>0.10)$. In addition, there were no significant results for scenes or objects when we examined the effects of threat and encoding success on PPA-LC functional connectivity $(p>0.05)$.
Whole-brain results. At the whole-brain level, we found that right PPA functional connectivity with early visual processing regions, including the left occipital fusiform gyrus and LOC, diminished significantly when participants successfully encoded scenes under threat (Fig. 6). This finding suggests that reduced PPA-LOC functional coupling, or potentially more competitive network-level interactions (i.e., anti-correlated functional connectivity), between high- and lower-priority visual cortex was associated with arousal-enhanced encoding of the goal-relevant scene stimulus. We did not find any other main nor interaction effects on right or left PPA-seeded functional connectivity, nor did we find any significant results for PPA functional connectivity associated with object encoding.

Relationship between individual differences in PPA functional connectivity and threat-related memory outcomes. Robust correlation analyses revealed that, under threat, greater right PPA-right LOC scene encoding functional connectivity was marginally anticorrelated with threat-impaired object memory $\left(\beta_{(13)}=-0.93\right.$, $p=0.06)$ and threat-enhanced memory scene memory $\left(\beta_{(13)}=\right.$ $-0.62, p=0.11$ ) (Table 6). Consistent with our main prediction, right PPA-right LOC gain-related patterns in functional connectivity were also significantly positively associated with greater gain effects in memory under threat $\left(\beta_{(17)}=-1.02, p=0.01\right.$; Fig. $6)$. This finding indicates that, under threat, individuals who showed more competitive (i.e., anticorrelated) PPA-LOC functional interactions when encoding high- versus lower-priority information also showed enhanced high-priority scene memory and impaired lower-priority object memory under threat. 
Table 5. Effects of threat of punishment on whole-brain parahippocampal place area (PPA) functional connectivity during successful scene encoding, as parametrically modulated by pupil dilation responses to the overlap image

\begin{tabular}{|c|c|c|c|c|c|c|}
\hline \multirow[b]{2}{*}{ Brain region } & \multirow[b]{2}{*}{ Cluster } & \multirow[b]{2}{*}{ Z-statistic } & \multicolumn{3}{|c|}{ MNI Peak Coordinates (mm) } & \multirow[b]{2}{*}{ Voxels } \\
\hline & & & $x$ & Y & $Z$ & \\
\hline \multicolumn{7}{|l|}{ Scene memory } \\
\hline \multicolumn{7}{|l|}{ Right PPA } \\
\hline Threat $>$ neutral & - & - & - & - & - & - \\
\hline Remember $>$ Forgot & - & - & - & - & - & - \\
\hline \multicolumn{7}{|l|}{ Neutral (Remember $>$ Forgot) - threat (Remember $>$ Forgot) } \\
\hline Right occipital pole & 2 & 3.62 & 10 & -92 & -20 & 652 \\
\hline Left occipital pole & 2 & 3.14 & -2 & -90 & -24 & \\
\hline Left occipital fusiform gyrus & 2 & 3.14 & -12 & -86 & -26 & \\
\hline Left occipital fusiform cortex & 2 & 3.12 & -12 & -84 & -22 & \\
\hline Left lingual gyrus & 2 & 3.05 & -4 & -90 & -20 & \\
\hline Left occipital fusiform gyrus & 2 & 3.04 & -12 & -90 & -20 & \\
\hline Left occipital fusiform gyrus & 1 & 3.16 & -42 & -70 & -24 & 397 \\
\hline Left occipital fusiform gyrus & 1 & 2.98 & -38 & -76 & -24 & \\
\hline Left lateral occipital cortex & 1 & 2.95 & -34 & -82 & -26 & \\
\hline Left occipital fusiform gyrus/LOC & 1 & 2.88 & -40 & -68 & -16 & \\
\hline Left occipital fusiform gyrus & 1 & 2.84 & -32 & -76 & -24 & \\
\hline Left temporal occipital fusiform cortex & 1 & 2.83 & -34 & -66 & -24 & \\
\hline \multicolumn{7}{|l|}{ Left PPA } \\
\hline Threat $>$ Neutral & - & - & - & - & - & - \\
\hline Remember $>$ Forgot & - & - & - & - & - & - \\
\hline Threat (Remember $>$ Forgot)-Neutral (Remember $>$ Forgot) & - & - & - & - & - & - \\
\hline \multicolumn{7}{|l|}{ Object memory } \\
\hline \multicolumn{7}{|l|}{ Right PPA } \\
\hline Threat $>$ neutral & - & - & - & - & - & - \\
\hline Remember $>$ Forgot & - & - & - & - & - & - \\
\hline Neutral (Remember $>$ Forgot) - threat (Remember $>$ Forgot) & - & - & - & - & - & - \\
\hline \multicolumn{7}{|l|}{ Left PPA } \\
\hline Threat $>$ neutral & - & - & - & - & - & - \\
\hline Remember $>$ Forgot & - & - & - & - & - & - \\
\hline Threat (Remember $>$ Forgot) - neutral (Remember $>$ Forgot) & - & - & - & - & - & - \\
\hline
\end{tabular}

For brainstem alignment, results are only reported if clusters were centered upon voxels in brainstem or midbrain, because this alignment procedure may lead to misalignment of higher subcortical and cortical structures.

H, Hemisphere; PHG, parahippocampal gyrus; TO, temporal occipital; R, remember; F, forgot.

\section{Discussion}

During arousing moments, the release of catecholamines is essential for forming adaptive memories of significant events (Harley, 2004). However, unlike the well established role of the dopaminergic system in facilitating reward-motivated learning (Sara, 2009), considerably less is known about the LC-NE system's contributions to motivated encoding processes, particularly when humans are faced with threat. Using both fMRI and pupil dilation measures, a noninvasive biomarker of phasic LC activity (Murphy et al., 2014; Joshi et al., 2016; Varazzani et al., 2015; Reimer et al., 2016; Liu et al., 2017), our results provide the first empirical support for the idea that a momentary increase in arousal engages the LC to selectively process and store prioritized information regardless of its intrinsic emotionality.

Our behavioral results revealed that threat-induced arousal amplified the effects of top-down priority such that memory for goal-relevant stimuli was selectively enhanced. Much work shows that motivation enhances declarative memory encoding and can narrow the focus of attention onto stimuli relevant to our goals (Levine and Edelstein, 2009; Kaplan et al., 2012; Montagrin et al., 2013), at least when rewarding or punishment-related incentives precede actual goal attainment (Kaplan et al., 2012). Consistent with this, prior studies show that cues associated with the threat of shock (Murty et al., 2012) or monetary punishment (Shigemune et al., 2014) lead to enhanced memory for neutral standalone scene and word images, respectively. By demonstrating that, like emotional stimuli, threat of punishment impacts memory of neutral representations differently depending on their goal relevance, our results are an important extension of prior work
(Mather and Sutherland, 2011; Sakaki et al., 2014; Lee et al., 2015). We also found a statistical trend toward the predicted threat-related impairment of low-priority stimuli. Low-priority memory was significantly above chance in the neutral condition, suggesting that participants were able to encode some distracting inputs. However, because performance in the threat condition was not significantly above chance, there may have been a floor effect that limited the range for a meaningful object memory comparison. Future studies could promote stronger encoding of both stimuli, such as increasing the image duration, to improve the ability to detect reliable threat-related memory impairments for less salient information.

Using trial-level pupil dilation as a proxy of LC activity (Murphy et al., 2014), we showed that enhanced threat-related pupil dilations specifically predicted better memory for goal-relevant images. In similar findings, previous work shows that greater pupil dilation to high-value words versus low-value words is associated with reward-related memory enhancements (Ariel and Castel, 2014). Likewise, pupil dilations evoked by events known to activate the LC-NE system, including target detection (Hoffing and Seitz, 2015) or oddball sounds (Tona et al., 2016), lead to enhanced encoding of concurrent scene images. However, to date, the notion that these effects signify LC neuromodulation has been limited to speculation because these studies did not measure LC activity directly in the brain. By weighting the BOLD response by pupil dilation, our imaging results support a link between pupil responses during memory encoding and human LC activity. Interestingly, pupil dilation has been shown to predict subsequent perceptual stability of one percept over another 


\begin{tabular}{|c|c|c|c|}
\hline Threat $(\mathrm{R}>\mathrm{F})>$ neutral $(\mathrm{R}>\mathrm{F}) \mathrm{ROI}$ connectivity ${ }^{\#}$ & Estimate & SE & $p$ \\
\hline \multicolumn{4}{|c|}{ Relationship with threat-related suppression of low-priority object memory } \\
\hline Intercept & -0.12 & 0.37 & 0.93 \\
\hline Right PPA-right LOC: scene encoding & -0.93 & 0.41 & $0.06 \sim$ \\
\hline Right PPA-left LOC: scene encoding & 0.70 & 0.55 & 0.51 \\
\hline Left PPA-LOC: scene encoding & 0.28 & 0.40 & 0.60 \\
\hline Left PPA-LOC: scene encoding & -0.54 & 0.41 & 0.49 \\
\hline Right PPA-right LOC: object encoding & 0.34 & 0.36 & 0.37 \\
\hline Right PPA-left LOC: object encoding & 0.04 & 0.37 & 0.85 \\
\hline Left PPA-right LOC: object encoding & -0.17 & 0.32 & 0.75 \\
\hline Left PPA-left LOC: object encoding & 0.26 & 0.38 & 0.41 \\
\hline \multicolumn{4}{|c|}{ Relationship with threat-related enhancement of high-priority scene memory } \\
\hline Intercept & -0.06 & 0.27 & 0.62 \\
\hline Right PPA-right LOC: scene encoding & -0.62 & 0.33 & $0.07 \sim$ \\
\hline Right PPA-left LOC: scene encoding & 0.10 & 0.55 & 0.79 \\
\hline Left PPA-right LOC: scene encoding & -0.08 & 0.36 & 0.73 \\
\hline Left PPA-left LOC: scene encoding & 0.67 & 0.44 & 0.17 \\
\hline Right PPA-right LOC: object encoding & 0.23 & 0.35 & 0.47 \\
\hline Right PPA-left LOC: object encoding & 0.10 & 0.39 & 0.70 \\
\hline Left PPA-right LOC: object encoding & -0.02 & 0.31 & 0.88 \\
\hline Left PPA-left LOC: object encoding & -0.39 & 0.38 & 0.44 \\
\hline \multicolumn{4}{|c|}{ Relationship with threat-enhanced gain effects in memory } \\
\hline Intercept & 0.01 & 0.26 & 0.98 \\
\hline Right PPA-right LOC: gain effect & -1.02 & 0.33 & $0.01^{*}$ \\
\hline Right PPA-left LOC: gain effect & 0.12 & 0.36 & 0.72 \\
\hline Left PPA-right LOC: gain effect & 0.36 & 0.29 & 0.27 \\
\hline Left PPA-left LOC: gain effect & 0.02 & 0.31 & 0.73 \\
\hline
\end{tabular}

"Gain" refers to a three-way interaction score between priority, memory and arousal: scene [threat $(R>F)$ neutral $(R>F)$ - object [threat $(R>F)-$ neutral $(R>F)$ ].

\#Functional connectivity estimate between PPA and LOC during encoding. $\sim p<0.10$; ${ }^{*} p<0.05$. $R$, remember; $F$, forgot

during perceptual rivalry (Einhäuser et al., 2008). From the perspective that phasic LC activity amplifies neural gain (AstonJones and Cohen, 2005; Mather et al., 2016), our results suggest that threat-related LC activity biases awareness to favor prioritized neutral representations.

Consistent with this, we found that LC activity was associated with more selective and localized neuronal processing under arousing conditions. Specifically, stimulus-evoked pupil dilations modulated scene-encoding activity in the LC and PHC, a predominantly scene-selective region (Köhler et al., 2002; Staresina et al., 2011). LC neuromodulation did not relate to scene encoding activity in the LOC (the region representing obects; Grill-Spector et al., 1999), indicating that such regulation is localized to prioritized representational cortex. In fact, our wholebrain analysis revealed that threat-induced arousal modulated scene-encoding activity in the LC/PHC and nowhere else. Unlike studies focused on perception (Lee et al., 2014), we observed pupil-modulated encoding patterns farther along the ventral visual stream than PPA in the PHC and posterior hippocampus, which also show some selectivity for scene encoding (Preston et al., 2010; Mundy et al., 2012). This finding is consistent with recent imaging studies showing that aversive contexts amplify PHC responses to surprising events (Murty et al., 2016) and during threat-motivated source memory binding (Shigemune et al., 2014). In sum, our results support a recent model positing that LC neuromodulation enhances processing important representations selectively (Mather et al., 2016).
Our whole-brain fMRI analyses also revealed that LOC activity was associated with successful object encoding under threat. It is important to stress that, at the behavioral level, threat trended toward significantly suppressing distracter encoding. Therefore, even though distracter encoding was uncommon, this result indicates that threat modulated visual cortex activity only when objects were successfully encoded. Although speculative, one possibility is that object inputs sometimes triggered greater stimulus-driven attention and reorienting, thereby becoming more prioritized. However, this threat-induced strengthening of object traces did not relate to LC activation, suggesting that other threat-related arousal mechanisms facilitate encoding of lowpriority representations.

Another interesting finding was that, across arousal conditions, successful scene encoding corresponded with reduced activity in the left frontal operculum/insula and left/right TPJ. Prior work implicates these regions in processing internal state information (Craig, 2009) and bottom-up reorienting (Corbetta and Shulman, 2002). Further, other neuroimaging work demonstrates that increased TPJ activity impairs episodic memory encoding of goal-relevant stimuli (Uncapher et al., 2011). Therefore, our findings are consistent with the idea that successful top-down encoding processes rely on the effective suppression of task-irrelevant internal and external information.

By simultaneously modeling pupil dilations to the monetary cue and overlap stimulus, we were also able to link the mnemonic benefit of putative LC activity to the stimulus-encoding period. Why would the LC's mnemonic influence be evident during the encoding event but not during the arousing cue? One possibility is that increased pupil dilation at encoding reflects more effortful cognition and energy investment (Kahneman and Beatty, 1966; Varazzani et al., 2015). Recent work in monkeys shows that, although pupil dilation occurs in responses to reward cues, LC activity was more closely correlated with dilations during the onset of goaldirected behavioral responses and the degree of effort requires to perform those actions (Varazzani et al., 2015). Therefore, our finding of a positive relationship between pupil-modulated LC encoding activity may signify participants' increased effort to encode target information, consistent with evidence implicating the LC in effortful, goal-directed attention, and behavior (Aston-Jones and Cohen, 2005; Raizada and Poldrack, 2007; Bouret et al., 2012; Alnæs et al., 2014; Floresco, 2015).

The notion that our pupil and LC activity results reflect increased attentional modulation rather than emotional arousal, per se, aligns with extant neurobiological models of emotional memory. Specifically, converging theoretical and empirical work demonstrate that the immediate benefit of attention on emotional memory are dissociable from longer-term emotional memory enhancements (Sharot and Phelps, 2004; Talmi et al., 2008, 2013), with the latter involving postencoding noradrenergic modulation of the amygdala (McGaugh and Roozendaal, 2002; LaLumiere et al., 2003). Although we cannot dissociate whether LC activation in this study corresponded with greater selective attention versus emotional arousal (or both), the GANE model is agnostic to how NE levels become elevated: insofar as the LC is sufficiently activated by a salient event, we would expect enhanced memory selectivity for information that is prioritized at that moment (Mather et al., 2016). Interestingly, empirical work shows that the facilitating effects of emotional arousal on prioritized neutral memoranda become even more pronounced after a 1-week delay (Anderson et al., 2006; Knight and Mather, 2009). Therefore, one avenue for future research would be to determine whether enhancing postencoding noradrenergic activity amplifies the priority-dependent ef- 
fects of phasic LC activation at encoding (Roozendaal and Hermans, 2017).

Prior research using neuromelanin-sensitive imaging has linked LC signal intensity, a structural marker of LC neuronal density (Keren et al., 2009, 2015), to trait measures of executive function (Clewett et al., 2016) and parasympathetic control over the heart (Mather et al., 2017). The present study expands upon these studies by demonstrating that individuals with greater LC neuromelanin are also more likely to engage the LC to encode task-relevant representations under arousal. To our knowledge, these data provide the first human evidence of a direct link between task-related LC activity and its structure. These results highlight the importance of measuring individual differences in LC structure because it may account for differences in cognitive abilities (Clewett et al., 2016), vulnerability to anxiety (Bangasser et al., 2016), relative levels of tonic sympathetic activity (Mather et al., 2017), or age-related neuronal pathology (Braak et al., 2011; Robertson, 2013; Mather and Harley, 2016).

Using functional connectivity analyses, we found that successful threat-motivated encoding yielded dynamic coupling patterns consistent with increased neural gain. Specifically, better high-priority encoding under threat was associated with reduced functional coupling between high-priority cortex (PPA) and lower-priority cortex (LOC). Further, an individual differences analysis revealed that, under threat, individuals who showed less communication between these competing visual regions when encoding high- versus lower-priority information showed more selective memory for goal-relevant information. Therefore, beyond influencing perception (Lee et al., 2014), arousal also seems to bias network-level processes to support "winner-take-more" and "loser-take-less" effects in memory. These results are consistent with prior theoretical (Aston-Jones and Cohen, 2005; Mather et al., 2016) and neural network models (Servan-Schreiber et al., 1990; Usher et al., 1999; Eldar et al., 2013, 2016) of LC gain modulation proposing that lateral inhibition between competing brain regions is critical for biasing processing in favor of task-relevant over taskirrelevant stimuli. Supporting this idea, a transcranial magnetic stimulation study showed that inhibiting LOC activity improves scene categorization accuracy via an increase in PPA activity (Mullin and Steeves, 2011, 2013). Therefore, effective distracter suppression may also liberate mental or energetic resources to focus even more on encoding the most important representations.

There are several limitations in our study. First, imaging human brainstem activity is often confounded by cardiac pulsation artifact, the LC's small size, and low spatial resolution of fMRI (Astafiev et al., 2010). Importantly, we used a rigorous methodological approach to mitigate these issues. A brainstem-optimized alignment procedure was used for image registration (Napadow et al., 2006) and we removed confounding signals in the fourth ventricle using ICA (Beckmann and Smith, 2004; Clewett et al., 2013). We also analyzed the task data using regressors modulated by trial-level pupil dilation as proxy for phasic LC activity. Finally, we used neuromelanin-sensitive weighted imaging to acquire participant-specific LC structural ROIs (Sasaki et al., 2006; Shibata et al., 2006; Clewett et al., 2016). The significant relationship between LC BOLD signal and LC neuromelanin signal helps to validate that our imaging results signified true LC activity. Moreover, it is unlikely that our findings resulted from threat amplifying physiological noise related to greater sympathetic arousal because we did not observe a main effect of threat on LC activity. Nonetheless, our results should be interpreted with caution given current methodological constraints on imaging human brains- tem activity. Another important consideration is that we paired aversive sounds with the threat cues to amp up overall arousal. Given that prior studies have reported greater VTA activity in response to only images of threatening money cues (Shigemune et al., 2014), it is possible that our null dopaminergic results may have been driven by a combination of both aversive motivational and unconditioned stressors.

In conclusion, our findings support the idea that LC activity helps to optimize the selection and formation of adaptive memories when it is most needed. Ultimately, this work will inform future studies investigating the role of neuromodulators in motivated cognition, particularly with respect to avoiding threat and energizing adaptive behavior. Furthermore, our results may inform how arousal-related disorders characterized by the formation of maladaptive memories under threat (e.g., PTSD) or inabilities to focus attention (e.g., ADHD) relate to altered LC-NE system function.

\section{References}

Adcock RA, Thangavel A, Whitfield-Gabrieli S, Knutson B, Gabrieli JD (2006) Reward-motivated learning: Mesolimbic activation precedes memory formation. Neuron 50:507-517. CrossRef Medline

Alnæs D, Sneve MH, Espeseth T, Endestad T, van de Pavert SHP, Laeng B (2014) Pupil size signals mental effort deployed during multiple object tracking and predicts brain activity in the dorsal attention network and the locus coeruleus. J Vis 14: pii: 1. CrossRef Medline

Anderson AK, Wais PE, Gabrieli JD (2006) Emotion enhances remembrance of neutral events past. Proc Natl Acad Sci U S A 103:1599-1604. CrossRef Medline

Ariel R, Castel A (2014) Eyes wide open: enhanced pupil dilation when selectively studying important information. Exp Brain Res 232:337-344. CrossRef Medline

Astafiev SV, Snyder AZ, Shulman GL, Corbetta M (2010) Comment on "Modafinil shifts human locus coeruleus to low-tonic, high-phasic activity during functional MRI" and "Homeostatic sleep pressure and responses to sustained attention in the suprachiasmatic area". Science 328 309. CrossRef Medline

Aston-Jones G, Cohen JD (2005) An integrative theory of locus coeruleusnorepinephrine function: adaptive gain and optimal performance. Annu Rev Neurosci 28:403-450. CrossRef Medline

Aston-Jones G, Rajkowski J, Cohen J (1999) Role of locus coeruleus in attention and behavioral flexibility. Biol Psychiatry 46:1309-1320. CrossRef Medline

Baayen, RH., Davidson, DJ., and Bates, D. M (2008) Mixed-effects modeling with crossed random effects for subjects and items. J Mem Lang 59: 390-412. CrossRef

Bangasser DA, Wiersielis KR, Khantsis S (2016) Sex differences in the locus coeruleus-norepinephrine system and its regulation by stress. Brain Res 1641:177-188. CrossRef Medline

Beckmann CF, Smith SM (2004) Probabilistic independent component analysis for functional magnetic resonance imaging. IEEE Trans Med Imaging 23:137-152. CrossRef Medline

Beckmann CF, Jenkinson M, Smith SM (2003) General multilevel linear modeling for group analysis in fMRI. Neuroimage 20:1052-1063. CrossRef Medline

Beckmann CF, DeLuca M, Devlin JT, Smith SM (2005) Investigations into resting-state connectivity using independent component analysis. Philos Trans R Soc Lond B Biol Sci 360:1001-1013. CrossRef Medline

Berridge CW, Waterhouse BD (2003) The locus coeruleus-noradrenergic system: modulation of behavioral state and state-dependent cognitive processes. Brain Res Rev 42:33-84. CrossRef Medline

Bisby JA, Burgess N (2013) Negative affect impairs associative memory but not item memory. Learn Mem 21:21-27. CrossRef Medline

Bouret S, Richmond BJ (2015) Sensitivity of locus ceruleus neurons to reward value for goal-directed actions. J Neurosci 35:4005-4014. CrossRef Medline

Bouret S, Ravel S, Richmond BJ (2012) Complementary neural correlates of motivation in dopaminergic and noradrenergic neurons of monkeys. Front Behav Neurosci 6:40. CrossRef Medline

Braak H, Thal DR, Ghebremedhin E, Del Tredici K (2011) Stages of the 
pathologic process in Alzheimer disease: age categories from 1 to 100 years. J Neuropathol Exp Neurol 70:960-969. CrossRef Medline

Bradley, M. M., and Lang, P. J (2007) The International Affective Digitized Sounds, Ed 2; IADS-2: Affective ratings of sounds and instruction manual, Technical Report B-3. Gainesville, FL: University of Florida.

Clewett D, Schoeke A, Mather M (2013) Amygdala functional connectivity is reduced after the cold pressor task. Cogn Affect Behav Neurosci 13:501518. CrossRef Medline

Clewett DV, Lee TH, Greening S, Ponzio A, Margalit E, Mather M (2016) Neuromelanin marks the spot: identifying a locus coeruleus biomarker of cognitive reserve in healthy aging. Neurobiol Aging 37:117-126. CrossRef Medline

Clewett D, Sakaki M, Huang R, Nielsen SE, Mather M (2017a) Arousal amplifies biased competition between high and low priority memories more in women than in men: The role of elevated noradrenergic activity. Psychoneuroendocrinology 80:80-91. CrossRef Medline

Clewett D, Sakaki M, Nielsen S, Petzinger G, Mather M (2017b) Noradrenergic mechanisms of arousal's bidirectional effects on episodic memory. Neurobiol Learn Mem 137:1-14. CrossRef Medline

Corbetta M, Shulman GL (2002) Control of goal-directed and stimulusdriven attention in the brain. Nat Rev Neurosci 3:201-215. Medline

Craig AD (2009) How do you feel-now? The anterior insula and human awareness. Nat Rev Neurosci 10:59-70. CrossRef Medline

Dunsmoor JE, Martin A, LaBar KS (2012) Role of conceptual knowledge in learning and retention of conditioned fear. Biol Psychol 89:300-305. CrossRef Medline

Dunsmoor JE, Murty VP, Davachi L, Phelps EA (2015) Emotional learning selectively and retroactively strengthens memories for related events. Nature 520:345-348. CrossRef Medline

Easterbrook, JA (1959) The effect of emotion on cue utilization and the organization of behavior. Psychol Rev 66:183-201. CrossRef Medline

Einhäuser W, Stout J, Koch C, Carter O (2008) Pupil dilation reflects perceptual selection and predicts subsequent stability in perceptual rivalry. Proc Natl Acad Sci U S A 105:1704-1709. CrossRef Medline

Eldar E, Niv Y, Cohen JD (2016) Do you see the forest or the tree? Neural gain and breadth versus focus in perceptual processing. Psychol Sci 27: 1632-1643. CrossRef Medline

Eldar E, Cohen JD, Niv Y (2013) The effects of neural gain on attention and learning. Nat Neurosci 16:1146-1153. CrossRef Medline

Epstein R, Kanwisher N (1998) A cortical representation of the local visual environment. Nature 392:598-601. CrossRef Medline

Floresco SB (2015) Noradrenaline and dopamine: sharing the workload. Trends Neurosci 38:465-467. CrossRef Medline

Friston KJ, Buechel C, Fink GR, Morris J, Rolls E, Dolan RJ (1997) Psychophysiological and modulatory interactions in neuroimaging. Neuroimage 6:218-229. CrossRef Medline

Gabrieli JD, Brewer JB, Desmond JE, Glover GH (1997) Seperate neural bases of two fundamental memory processes in the human medial temporal lobe. Science 276:264-266. CrossRef Medline

Grill-Spector K, Kushnir T, Edelman S, Avidan G, Itzchak Y, Malach R (1999) Differential processing of objects under various viewing conditions in the human lateral occipital complex. Neuron 24:187-203. CrossRef Medline

Harley CW (2004) Norepinephrine and dopamine as learning signals. Neural Plast 11:191-204. CrossRef Medline

Hoffing RC, Seitz AR (2015) Pupillometry as a glimpse into the neurochemical basis of human memory encoding. J Cogn Neurosci 27:765-774. CrossRef Medline

Hurlemann R, Hawellek B, Matusch A, Kolsch H, Wollersen H, Madea B, Vogeley K, Maier W, Dolan RJ (2005) Noradrenergic modulation of emotion-induced forgetting and remembering. J Neurosci 25:63436349. CrossRef Medline

Joshi S, Li Y, Kalwani RM, Gold JI (2016) Relationships between pupil diameter and neuronal activity in the locus coeruleus, colliculi, and cingulate cortex. Neuron 89:221-234. CrossRef Medline

Kahneman D, Beatty J (1966) Pupil diameter and load on memory. Science 154:1583-1585. CrossRef Medline

Kaplan RL, Van Damme I, Levine LJ (2012) Motivation matters: Differing effects of pre-goal and post-goal emotions on attention and memory. Front Psychol 3:404. CrossRef Medline

Kensinger EA, Garoff-Eaton RJ, Schacter DL (2007) How negative emotion enhances the visual specificity of a memory. J Cogn Neurosci 19:18721887. CrossRef Medline

Kensinger, E. A., Garoff-Eaton, R. J., and Schacter, D. L (2006) Memory for specific visual details can be enhanced by negative arousing content. J Mem Lang 54:99-112. CrossRef

Keren NI, Taheri S, Vazey EM, Morgan PS, Granholm AC, Aston-Jones GS, Eckert MA (2015) Histologic validation of locus coeruleus MRI contrast in post-mortem tissue. Neuroimage 113:235-245. CrossRef Medline

Keren NI, Lozar CT, Harris KC, Morgan PS, Eckert MA (2009) In vivo mapping of the human locus coeruleus. Neuroimage 47:1261-1267. CrossRef Medline

Knight M, Mather M (2009) Reconciling findings of emotion-induced memory enhancement and impairment of preceding items. Emotion 9:763-781. CrossRef Medline

Knutson B, Westdorp A, Kaiser E, Hommer D (2000) fMRI visualization of brain activity during a monetary incentive delay task. Neuroimage 12:20 27. CrossRef Medline

Köhler S, Crane J, Milner B (2002) Differential contributions of the parahippocampal place area and the anterior hippocampus to human memory for scenes. Hippocampus 12:718-723. CrossRef Medline

LaBar KS, Cabeza R (2006) Cognitive neuroscience of emotional memory. Nat Rev Neurosci 7:54-64. CrossRef Medline

LaLumiere RT, Buen TV, McGaugh JL (2003) Post-training intra-basolateral amygdala infusions of norepinephrine enhance consolidation of memory for contextual fear conditioning. J Neurosci 23:6754-6758. Medline

Lee TH, Sakaki M, Cheng R, Velasco R, Mather M (2014) Emotional arousal amplifies the effects of biased competition in the brain. Soc Cogn Affect Neurosci 9:2067-2077. CrossRef Medline

Lee TH, Greening SG, Mather M (2015b) Encoding on goal-relevant stimuli is strengthened by emotional stimuli in memory. Front Psychol 6:1173. CrossRef Medline

Levine LJ, Edelstein RS (2009) Emotion and memory narrowing: a review and goal-relevance approach. Cognition and Emotion 23:833-875. CrossRef

Liu Y, Rodenkirch C, Moskowitz N, Schriver B, Wang Q (2017) Dynamic lateralization of pupil dilation evoked by locus coeruleus activation results from sympathetic, not parasympathetic, contributions. Cell Rep 20: 3099-3112. CrossRef Medline

Markovic J, Anderson AK, Todd RM (2014) Tuning to the significant: Neural and genetic processes underlying affective enhancement of visual perception and memory. Behav Brain Res 259:229-241. CrossRef Medline

Mather M, Harley CW (2016) The locus coeruleus: Essential for maintaining cognitive function and the aging brain. Trends Cogn Sci 20:214-226. CrossRef Medline

Mather M, Sutherland MR (2011) Arousal-biased competition in perception and memory. Perspect Psychol Sci 6:114-133. CrossRef Medline

Mather M, Clewett D, Sakaki M, Harley CW (2016) Norepinephrine ignites local hot spots of neuronal excitation: how arousal amplifies selectivity in perception and memory. Behav Brain Sci 39:e200. CrossRef Medline

Mather M, Joo Yoo H, Clewett DV, Lee TH, Greening SG, Ponzio A, Min J, Thayer JF (2017) Higher locus coeruleus MRI contrast is associated with lower parasympathetic influence over heart rate variability. Neuroimage 150:329-335. CrossRef Medline

McGaugh JL (2013) Making lasting memories: remembering the significant. Proc Natl Acad Sci U S A 110:10402-10407. CrossRef Medline

McGaugh JL, Roozendaal B (2002) Role of adrenal stress hormones in forming lasting memories in the brain. Curr Opin Neurobiol 12:205-210. CrossRef Medline

Mohanty A, Gitelman DR, Small DM, Mesulam MM (2008) The spatial attention network interacts with limbic and monoaminergic systems to modulate motivation-induced attention shifts. Cereb Cortex 18:26042613. CrossRef Medline

Montagrin A, Brosch T, Sander D (2013) Goal conduciveness as a key determinant of memory facilitation. Emotion 13:622-628. CrossRef Medline

Mullin CR, Steeves JK (2011) TMS to the lateral occipital cortex disrupts object processing but facilitates scene processing. J Cogn Neurosci 23: 4174-4184. CrossRef Medline

Mullin CR, Steeves JK (2013) Consecutive TMS-fMRI reveals an inverse relationship in BOLD signal between object and scene processing. J Neurosci 33:19243-19249. CrossRef Medline

Mundy ME, Downing PE, Graham KS (2012) Extrastriate cortex and medial temporal lobe regions respond differentially to visual feature overlap within 
preferred stimulus category. Neuropsychologia 50:3053-3061. CrossRef Medline

Murphy PR, O'Connell RG, O'Sullivan M, Robertson IH, Balsters JH (2014) Pupil diameter covaries with BOLD activity in human locus coeruleus. Hum Brain Mapp 35:4140-4154. CrossRef Medline

Murphy PR, Robertson IH, Balsters JH, O'connell RG (2011) Pupillometry and $\mathrm{P} 3$ index the locus coeruleus-noradrenergic arousal function in humans. Psychophysiology 48:1532-1543. CrossRef Medline

Murty VP, Labar KS, Adcock RA (2012) Threat of punishment motivates memory encoding via amygdala, not midbrain, interactions with the medial temporal lobe. J Neurosci 32:8969-8976. CrossRef Medline

Murty VP, Shermohammed M, Smith DV, Carter RM, Huettel SA, Adcock RA (2014) Resting state networks distinguish human ventral tegmental area from substantia nigra. Neuroimage 100:580-589. CrossRef Medline

Murty VP, LaBar KS, Adcock RA (2016) Distinct medial temporal networks encode surprise during motivation by reward versus punishment. Neurobiol Learn Mem 134:55-64. CrossRef Medline

Napadow V, Dhond R, Kennedy D, Hui KK, Makris N (2006) Automated brainstem co-registration (ABC) for MRI. Neuroimage 32:1113-1119. CrossRef Medline

Phelps EA, Ling S, Carrasco M (2006) Emotion facilitates perception and potentiates the perceptual benefits of attention. Psychol Sci 17:292-299. CrossRef Medline

Preston AR, Bornstein AM, Hutchinson JB, Gaare ME, Glover GH, Wagner AD (2010) High-resolution fMRI of content-sensitive subsequent memory responses in human medial temporal lobe. J Cogn Neurosci 22:156-173. CrossRef Medline

Raizada RD, Poldrack RA (2007) Challenge-driven attention: interacting frontal and brainstem systems. Front Hum Neurosci 1:3. CrossRef Medline

Reimer J, McGinley MJ, Liu Y, Rodenkirch C, Wang Q, McCormick DA, Tolias AS (2016) Pupil fluctuations track rapid changes in adrenergic and cholinergic activity in cortex. Nat Commun 7:13289. CrossRef Medline

Robertson IH (2013) A noradrenergic theory of cognitive reserve: implications for Alzheimer's disease. Neurobiol Aging 34:298-308. CrossRef Medline

Roozendaal B, Hermans EJ (2017) Norepinephrine effects on the encoding and consolidation of emotional memory: improving synergy between animal and human studies. Curr Opin Behav Sci 14:115-122. CrossRef

Sakaki M, Fryer K, Mather M (2014) Emotion strengthens high-priority memory traces but weakens low priority memory traces. Psychol Sci 25: 387-395. CrossRef Medline

Sara SJ (2009) The locus coeruleus and noradrenergic modulation of cognition. Nat Rev Neurosci 10:211-223. CrossRef Medline

Sasaki M, Shibata E, Tohyama K, Takahashi J, Otsuka K, Tsuchiya K, Takahashi S, Ehara S, Terayama Y, Sakai A (2006) Neuromelanin magnetic resonance imaging of locus ceruleus and substantia nigra in Parkinson's disease. Neuroreport 17:1215-1218. CrossRef Medline

Schwarze U, Bingel U, Sommer T (2012) Event-related nociceptive arousal enhances memory consolidation for neutral scenes. J Neurosci 32:14811487. CrossRef Medline

Servan-Schreiber D, Printz H, Cohen JD (1990) A network model of catecholamine effects: gain, signal-to-noise ratio, and behavior. Science 249: 892-895. CrossRef Medline

Sharot T, Phelps EA (2004) How arousal modulates memory: Disentangling the effects of attention and retention. Cogn Affect Behav Neurosci 4:294306. CrossRef Medline
Shibata E, Sasaki M, Tohyama K, Kanbara Y, Otsuka K, Ehara S, Sakai A (2006) Age-related changes in locus ceruleus on neuromelanin magnetic resonance imaging at 3 Tesla. Magnetic Resonance in Medical Sciences 5:197-200. CrossRef Medline

Shigemune Y, Tsukiura T, Kambara T, Kawashima R (2014) Remembering with gains and losses: effects of monetary reward and punishment on successful encoding activation of source memories. Cereb Cortex 24: 1319-1331. CrossRef Medline

Shohamy D, Adcock RA (2010) Dopamine and adaptive memory. Trends Cogn Sci 14:464-472. CrossRef Medline

Staresina BP, Duncan KD, Davachi L (2011) Perirhinal and parahippocampal cortices differentially contribute to later recollection of object-and scene-related event details. J Neurosci 31:8739-8747. CrossRef Medline

Stark CE, Squire LR (2001) When zero is not zero: The problem of ambiguous baseline conditions in fMRI. Proc Natl Acad Sci U S A 98:1276012766. CrossRef Medline

Strange BA, Dolan RJ (2004) beta-Adrenergic modulation of emotional memory-evoked human amygdala and hippocampal responses. Proc Natl Acad Sci U S A 101:11454-11458. CrossRef Medline

Strange BA, Hurlemann R, Dolan RJ (2003) An emotion-induced retrograde amnesia in humans is amygdala- and beta-adrenergic-dependent. Proc Natl Acad Sci U S A 100:13626-13631. CrossRef Medline

Sutherland MR, Mather M (2012) Negative arousal amplifies the effects of saliency in short-term memory. Emotion 12:1367-1372. CrossRef Medline

Talmi D (2013) Enhanced emotional memory: cognitive and neural mechanisms. Curr Dir Psychol Sci 22:430-436. CrossRef

Talmi D, Anderson AK, Riggs L, Caplan JB, Moscovitch M (2008) Immediate memory consequences of the effect of emotion on attention to pictures. Learn Mem 15:172-182. CrossRef Medline

Tona KD, Murphy PR, Brown SB, Nieuwenhuis S (2016) The accessory stimulus effect is mediated by phasic arousal: a pupillometry study. Psychophysiology 53:1108-1113. CrossRef Medline

Uncapher MR, Hutchinson JB, Wagner AD (2011) Dissociable effects of top-down and bottom-up attention during episodic encoding. J Neurosci 31:12613-12628. CrossRef Medline

Usher M, Cohen JD, Servan-Schreiber D, Rajkowski J, Aston-Jones G (1999) The role of locus coeruleus in the regulation of cognitive performance. Science 283:549-554. CrossRef Medline

Varazzani C, San-Galli A, Gilardeau S, Bouret S (2015) Noradrenaline and dopamine neurons in the reward/effort trade-off: a direct electrophysiological comparison in behaving monkeys. J Neurosci 35:7866-7877. CrossRef Medline

Weymar M, Bradley MM, Hamm AO, Lang PJ (2013) When fear forms memories: Threat of shock and brain potentials during encoding and recognition. Cortex 49:819-826. CrossRef Medline

Wilcox RR (2004) Some results on extensions and modifications of the Theil-Sen regression estimator. British Journal of Mathematical and Statistical Psychology 57:265-280. CrossRef Medline

Willenbockel V, Sadr J, Fiset D, Horne GO, Gosselin F, Tanaka JW (2010) Controlling low-level image properties: the SHINE toolbox. Behav Res Methods 42:671-684. CrossRef Medline

Worsley KJ (2001) Statistical analysis of activation images. In Functional MRI: an introduction to methods (Jezzard P, Matthews PM, Smith SM, eds). New York, NY: OUP. 